\title{
A CATALOG OF OUTER EJECTA KNOTS IN THE CASSIOPEIA A SUPERNOVA REMNANT ${ }^{1}$
}

\author{
Molly C. Hammell and Robert A. Fesen \\ Department of Physics and Astronomy, 6127 Wilder Lab, Dartmouth College, Hanover, NH 03755 \\ Received 2008 May 16; accepted 2008 June 27
}

\begin{abstract}
Hubble Space Telescope images of the core-collapse supernova remnant Cassiopeia A are used to identify highvelocity knots of ejecta located outside the remnant's main emission shell of expanding debris. These ejecta fragments are found near or ahead of the remnant's forward shock front and mostly lie from $120^{\prime \prime}$ to $300^{\prime \prime}$ in radial distance from the remnant's center of expansion. Filter flux ratios when correlated with published spectra show that these knots can be divided into three emission classes: (1) knots dominated by [N II] $\lambda \lambda 6548,6583$ emissions, (2) knots dominated by [O II] $\lambda \lambda 7319,7330$ emissions, and (3) knots displaying filter flux ratios suggestive of [S II], [O II], and [Ar III] $\lambda 7135$ emission line strengths similar to the "fast-moving knots" (FMKs) found in the remnant's bright main shell. Of 1825 knots identified, 444 are strong [ $\mathrm{N}$ II] emission knots, 192 are strong [O II] emission knots, and 1189 are FMK-like knots. In terms of location around the remnant, 972, 207, and 646 knots are found in the remnant's northeast jet, southwest jet, and non-jet regions, respectively. Assuming a distance of $3.4 \mathrm{kpc}$, derived knot transverse velocities based on proper motion measurements spanning a 9 month interval indicate maximum transverse expansion velocities for these three knot classes of 14,500,13,500, and 11,500 $\mathrm{km} \mathrm{s}^{-1}$, respectively. We present a catalog of these outlying ejecta clumps comprising finding charts, epoch 2004.2 knot positions, proper motions, photometric filter fluxes, and estimated knot emission type, along with cross-references to previous knot identifications and data. This compilation represents a nearly tenfold increase in the number of outlying, high-velocity ejecta knots identified around the Cassiopeia A remnant.
\end{abstract}

Subject headings: ISM: individual (Cassiopeia A) — ISM: kinematics and dynamics — supernova remnants

Online material: extended figure sets, machine-readable table

\section{INTRODUCTION}

Baade \& Minkowski (1954) were the first to identify optical emission associated with the bright Galactic radio source and young supernova remnant, Cassiopeia A (Cas A). Subsequent optical studies showed the presence of two distinct emission-line components. The bulk of the emission consists of a -4000 to $+6000 \mathrm{~km} \mathrm{~s}^{-1}$ expanding shell of knots, condensations, and filaments emitting strongly in [S II] $\lambda \lambda 6716,6731$ and $[\mathrm{O}$ III] $\lambda \lambda 4959,5007$ but showing no $\mathrm{H} \alpha$ emission. A few dozen semistationary condensations show strong $\mathrm{H} \alpha$ and [ $\mathrm{N}$ II] $\lambda \lambda 6548,6583$ emissions and prevalently negative radial velocities of $0-200 \mathrm{~km} \mathrm{~s}^{-1}$ (Minkowski 1957, 1968; van den Bergh \& Dodd 1970; van den Bergh 1971). These two chemically and dynamically distinct components became known as the remnant's fast-moving knots (FMKs) and quasi-stationary flocculi (QSFs), and are generally interpreted as high-velocity, undiluted SN ejecta and low-velocity pre-SN, circumstellar mass-loss material, respectively (van den Bergh 1971; Chevalier \& Kirshner 1978, 1979).

In even the earliest optical studies, a few emission knots could be seen outside of the main shell's $\simeq 2^{\prime}$ radius of FMKs. With the exception of a seemingly isolated knot along the remnant's northern edge (knot 91; Kamper \& van den Bergh 1976) and a few unusually distant QSFs situated along the remnant's southwestern limb (Kamper \& van den Bergh 1983), all of the remnant's outlying emission was concentrated in a "flare" (Minkowski 1957) or "jet" (van den Bergh \& Dodd 1970) along the remnant's northeastern (NE) limb, which extended out about $3.8^{\prime}$ from the remnant center at a position angle of around $65^{\circ}$. While the

\footnotetext{
${ }^{1}$ Based on observations with the NASA/ESA Hubble Space Telescope, obtained at the Space Telescope Science Institute, which is operated by the Association of Universities for Research in Astronomy, Inc. under NASA contract No. NAS5-26555.
}

origin and significance of these outer ejecta knots was unknown, early observations showed that this broad stream of ejecta off the remnant's $\mathrm{NE}$ limb could be readily imaged in [S II] but not [O III] emission. This fact, together with the jet's greater radial extent, implying greater expansion velocity, led to suggestions that it represented the lone surviving, undecelerated part of the supernova's highest velocity outer ejecta shell, and was chemically distinct from other FMKs in the main shell (Minkowski 1968; van den Bergh 1971).

The nature and distribution of outer ejecta fragments in the Cas A remnant became more complex when some 50 fast-moving $\left(v_{\text {trans }} \simeq 10,000 \mathrm{~km} \mathrm{~s}^{-1}\right)[\mathrm{N} \mathrm{II}]$ emission knots were discovered around the remnant's periphery (Fesen et al. 1987; Fesen 2001). One of these N-rich knots turned out to be knot 91, originally believed to be [S II] $\lambda \lambda 6716,6731$ bright, but in fact actually emits mainly [N II] $\lambda \lambda 6548,6583$ emission with an [ $\mathrm{N}_{\text {II] }}$ 6583/ $\mathrm{H} \alpha$ emission ratio $\simeq 30$. It was misidentified due to its detection on [S II] $\lambda \lambda 6716,6731$ images as a result of its $+4500 \mathrm{~km} \mathrm{~s}^{-1}$ radial velocity, which redshifts its [N II] $\lambda 6583$ line some $100 \AA$, thus placing it within the passband of a broad [S II] filter (van den Bergh \& Dodd 1970; Kamper \& van den Bergh 1976; van den Bergh \& Kamper 1985). This and other fast-moving, seemingly N-rich knots were interpreted as fragments from the Cas A progenitor's $\mathrm{N}$ and He-rich outer layers (Fesen et al. 1987).

Over 100 FMKs have now been identified in the NE jet, which extend out to radial distances of more than $2^{\prime}$ beyond the main shell, corresponding to transverse velocities approaching $15,000 \mathrm{~km} \mathrm{~s}^{-1}$ (Fesen \& Gunderson 1996), assuming a remnant distance of $3.4 \mathrm{kpc}$ (Reed et al. 1995). More recently, about a dozen FMKs have been discovered in an apparent southwest "counterjet" (Fesen 2001) weakly detected in X-rays and the infrared (Hwang et al. 2004; Hines et al. 2004; Ennis et al. 2006). These FMK-like ejecta in the NE and SW jets suggest a possibly 
TABLE 1

HST ACS Observations and Detected Line Emissions

\begin{tabular}{|c|c|c|c|c|c|c|}
\hline $\begin{array}{l}\text { ACS } \\
\text { Filter }\end{array}$ & $\begin{array}{l}\text { Exposure } \\
\text { Times }^{\mathrm{a}} \\
\text { (s) }\end{array}$ & $\begin{array}{c}\text { Filter } \\
\text { Bandpass }^{\mathrm{b}}\end{array}$ & $\begin{array}{c}\text { Main Line } \\
\text { Emissions in } \\
\text { Filter Bandpass }\end{array}$ & $\begin{array}{l}\text { ACS Filter } \\
\text { Throughput }\end{array}$ & $\begin{array}{c}\text { Relative } \\
\text { Observed } \\
\text { FMK Flux }\end{array}$ & $\begin{array}{l}\text { ACS Filter } \\
\text { FMK Flux }\end{array}$ \\
\hline $\begin{array}{l}\text { F625W } \\
(\mathrm{SDSS} r)\end{array}$ & $4 \times 600$ & $5450-7100$ & $\begin{array}{l}{\left[\mathrm{O}_{\mathrm{I}}\right] 6300,6364} \\
{[\mathrm{~S} \text { II }] 6716,6731} \\
{\left[\mathrm{~N}_{\text {II }}\right] 6548,6583}\end{array}$ & $\begin{array}{l}0.42 \\
0.44 \\
0.43\end{array}$ & $\begin{array}{r}39 \\
100 \\
0\end{array}$ & $\begin{array}{r}16 \\
44 \\
0\end{array}$ \\
\hline $\begin{array}{l}\text { F775W } \\
(\mathrm{SDSS} i)\end{array}$ & $4 \times 500$ & $6850-8600$ & $\begin{array}{l}{[\text { Ar III }] 7136} \\
{[\mathrm{O} \text { II }] 7319,7320} \\
{[\mathrm{Ar} \text { III }] 7751}\end{array}$ & $\begin{array}{l}0.38 \\
0.42 \\
0.38\end{array}$ & $\begin{array}{r}41 \\
170 \\
14\end{array}$ & $\begin{array}{r}15 \\
72 \\
5\end{array}$ \\
\hline 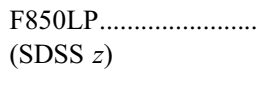 & $4 \times 500$ & 8100 & $\begin{array}{l}{[\mathrm{S} \text { III] } 9069} \\
{\left[\mathrm{S}_{\text {III }}\right] 9531} \\
{\left[\mathrm{~S}_{\text {II }}\right] 10287-10370}\end{array}$ & $\begin{array}{l}0.20 \\
0.14 \\
0.05\end{array}$ & $\begin{array}{l}210 \\
600 \\
380\end{array}$ & $\begin{array}{l}42 \\
85 \\
19\end{array}$ \\
\hline
\end{tabular}

${ }^{\text {a }}$ Exposure times are for all six remnant imaging fields.

${ }^{\mathrm{b}}$ Listed wavelengths represent approximate filter 5\% transmission cut-on and cutoff points in $\AA$. F850LP has a cutoff beyond $10500 \AA$.

${ }^{\mathrm{c}}$ Cas A FMK ejecta spectrum (FMK2; Hurford \& Fesen 1996). Values are relative to $F([\mathrm{~S}$ II $] 6716,6731)=100$.

asymmetric bipolar expansion of the Cas A supernova event (Fesen 2001). Evidence in favor of this interpretation is the detection only in the NE and SW jet regions of several so-called "mixed emission" knots, which exhibit strong emission lines of nitrogen, sulfur, and oxygen (Fesen \& Becker 1991; Fesen 2001).

The distribution and chemical abundances of the highest velocity debris can provide useful information regarding the explosion dynamics and ejecta mixing of the Cas A supernova event. In this paper, we present a more complete catalog of outlying optical ejecta knots in Cas A than previous surveys by using deep, multiband, Hubble Space Telescope images of the remnant. Partial analyses of data in this catalog have already been presented in Fesen et al. (2006a, 2006b). The catalog includes finding charts, epoch 2004.2 knot positions, proper motions, photometric filter fluxes, estimated knot emission type, and cross-references to previous knot identifications and data, along with some additional analysis of these data. The observations are described in $\S 2$, results presented in $\S 3$, with a short discussion given in $\S 4$.

\section{OBSERVATIONS}

High-resolution, multiband images of the Cas A remnant were obtained on 2004 March 4-6 and December 4-5 using the Wide Field Channel (WFC) of the Advanced Camera for Surveys (ACS; Ford et al. 1998; Pavlovsky et al. 2004) on board the Hubble Space Telescope $(H S T)$. The ACS/WFC consists of two $4096 \times 4096$ CCDs with an average pixel size of $0.05^{\prime \prime}$, providing a $202^{\prime \prime} \times 202^{\prime \prime}$ field of view.

Images were taken in the four Sloan Digital Sky Survey (SDSS) filters F450W, F625W, F775W, and F850LP $(g, r, i$, and $z$ ) at six target pointings covering the entire remnant, including all known outlying ejecta knots. Total integration times in each filter were $2000,2400,2000$, and $2000 \mathrm{~s}$, respectively. To remove cosmic ray hits, cover the $2.5^{\prime \prime}$ interchip gap, and minimize saturation effects of bright stars, a two-point ACS-WFC dither line (ACS Instrument Handbook; Boffi et al. 2003) was used, with two exposures taken at each dither point for the six pointings in each of the four filters.

Due to the significant amount of reddening toward Cas $\mathrm{A}\left(A_{V}=\right.$ 4.5-8 mag; Hurford \& Fesen 1996; Hwang \& Laming 2003; Reynoso \& Goss 2002), [O III] $\lambda \lambda 4959,5007$ was too weak to be detected for most outlying knots, and we were unable to include F450W images in our analysis. Primary emission lines detected for the remnant's ejecta using the three remaining filters (F625W, F775W, and F850LP) are listed in Table 1, along with the total system throughput of the telescope+ACS/WFC camera+filter, and the resulting relative line flux of a typical Cas A FMK ejecta knot.

Standard pipeline data reduction was performed using IRAF/ STSDAS. ${ }^{2}$ This included debiasing, flat-fielding, geometric distortion corrections, photometric calibrations, and cosmic ray and hot pixel removal. The STSDAS drizzle task was also used to combine single exposures in each filter.

Detected counts in each of the drizzled filter images were converted to flux units by summing the signal in $5 \times 5$ pixel windows, subtracting a local mean background, and then multiplying by the mean flux density per unit wavelength, generating 1 count $\mathrm{s}^{-1}$ (i.e., the "PHOTFLAM" factor) times the filter effective bandwidth (EBW). The PHOTFLAM value in units of $10^{-19} \mathrm{erg} \mathrm{cm}^{-2}$ $\mathrm{s}^{-1} \AA^{-1}$ and the EBW values used for the F625W, F775W, and F850LP filters were 1.195, 1.007, 1.507, and 415.5 ̊, 434.6 and $539.4 \AA$, respectively (Sirianni et al. 2005). Multiplying PHOTFLAM numbers by the EBW values leads to flux calibrations parameters of $4.97 \times 10^{-17}, 4.37 \times 10^{-17}$, and $8.13 \times 10^{-17}$ for filters F625W, F775W, and F850LP, respectively.

Calibrated estimates for fluxes of each outer optical ejecta knot in the three filters shown in Table 1 were calculated using the SExtractor (Bertin \& Arnouts 1996) automated source extraction software package. In cases where the SExtractor program failed to return a reasonable flux or failed to return a flux at all, knot fluxes were calculated by hand. Such manually measured filter fluxes were made for about 300 knots, with fluxes that were calculated using 5 pixel wide apertures. Background estimates were performed, by SExtractor, using a 24 pixel rectangular annulus about the isophotal limits of the object. When the object fluxes were calculated by hand, background estimation was performed by calculating the total 5 pixel aperture flux in at least five positions near the object (avoiding other sources) and then subtracting the mean computed "background" flux from the total object pixel sum. Most knots whose fluxes required manual computation were located near a bright background source or very close to another ejecta knot.

Flux estimates were best for the brightest, well-resolved knots (above $5 \times 10^{-16} \mathrm{erg} \mathrm{cm}^{-2} \mathrm{~s}^{-1}$ ), where errors are on the $5 \%-$ $10 \%$ level. Moderately bright knots $\left[(5-50) \times 10^{-17} \mathrm{erg} \mathrm{cm}^{-2} \mathrm{~s}^{-1}\right]$

\footnotetext{
2 IRAF is distributed by the National Optical Astronomy Observatories, which is operated by the Association of Universities for Research in Astronomy, Inc. (AURA), under cooperative agreement with the National Science Foundation. The Space Telescope Science Data Analysis System (STSDAS) is distributed by the Space Telescope Science Institute.
} 
suffered more from local background variations and uncertainties in knot positions, resulting in flux errors near $20 \%-30 \%$. Error estimates for faint knots $\left[(1-50) \times 10^{-18} \mathrm{erg} \mathrm{cm}^{-2} \mathrm{~s}^{-1}\right]$ lie approximately at the $50 \%$ level. Fainter knots, or nondetections in one of the filters, were set to the mean background levels of each filter as $\left[\sigma_{\text {back }}(\mathrm{F} 625 \mathrm{~W})=3.9 \times 10^{-19} \mathrm{erg} \mathrm{cm}^{-2} \mathrm{~s}^{-1}\right.$, $\sigma_{\text {back }}(\mathrm{F} 775 \mathrm{~W})=3.7 \times 10^{-19} \mathrm{erg} \mathrm{cm}^{-2} \mathrm{~s}^{-1}, \sigma_{\text {back }}(\mathrm{F} 850 \mathrm{~W})=$ $5.7 \times 10^{-19} \mathrm{erg} \mathrm{cm}^{-2} \mathrm{~s}^{-1}$ ] for the purposes of determining knot type by dominant emission features. Knots in this catalog were required to have a reasonable detection in at least one of the filters.

\section{RESULTS}

A comparison of 2004 March and December ACS/WFC images of Cas A revealed hundreds of compact optical emission knots around the remnant's outer periphery, the majority of which were previously unknown. We identify an outer knot, as opposed to a main shell knot, by (1) its location near or beyond the remnant's forward shock front emission as seen in X-ray images (Gotthelf et al. 2001; DeLaney \& Rudnick 2003; Hwang et al. 2004), (2) a small, compact appearance on the ACS/WFC images, and (3) a proper motion $\geq 0.30^{\prime \prime}$. No formal lower radial distance from the remnant's expansion center was set, but nearly all (99\%) of the knots identified lie out beyond a distance of $120^{\prime \prime}$, corresponding to the outer edge of the remnant's main emission shell (Gotthelf et al. 2001).

A total of 1825 outer knots were identified on these ACS/ WFC images. This number compares to previous studies which identified 100 NE knots in the remnant's northeast "jet," plus another 75 knots found elsewhere around the remnant (Fesen \& Gunderson 1996; Fesen 2001). Thus, this compilation represents a nearly tenfold increase in the number of known high-velocity outer ejecta knots in the remnant.

\subsection{Outlying Knot Emission Types}

It has been known for nearly two decades that there are at least two different populations of outer ejecta knots in Cas A. The first type could be recognized in even the earliest images taken of the remnant and is associated with the NE jet. These outer ejecta knots show strong $[\mathrm{S}$ II] $\lambda \lambda 6716,6731$ emission, much like that seen in the remnant's main shell (Fesen \& Gunderson 1996). The second type was discovered in 1987 and exhibits strong [ $\mathrm{N} \mathrm{II]}$ 226548, 6583 line emission (Fesen et al. 1987). These knots are mainly found outside of the NE jet region and display transverse expansion velocities comparable to many NE jet knots (see discussion and representative spectra in Fesen 2001).

An early result from our HST ACS imaging survey was the discovery of a substantial number of outlying knots which comprise a third class of outer, high-velocity debris. These have their 6000-10500 Å spectrum dominated by strong [O II] $\lambda \lambda 7319$, 7330 line emission and are presumably O-rich ejecta debris (Fesen et al. 2006a). This led to the realization that not only was there a far larger population of outlying high-velocity ejecta knots, but Cas A's outer ejecta possessed a broader range of emission line properties and likely chemical properties than previously realized.

When correlated with published optical spectra of outer knots, one can use the ACS/WFC images of Cas A taken in three SDSS filters to distinguish these three main types of outer emission knots based on filter fluxes (see Fesen et al. 2006a for a detailed discussion). In broad terms, outer knots either show an optical spectrum dominated by $[\mathrm{N}$ II] $\lambda \lambda 6548,6583$ emission or [O II] $\lambda \lambda 7319$, 7330 emission, or instead are like the NE jet knots and exhibit strong emission of [S II], [O II], and [Ar III] 27135 , similar to that seen to the remnant's main shell fast-moving knots (FMKs).
The ability of SDSS filter images to distinguish these three types of emission knots is shown in Table 1. The table lists the throughputs of the ACS/WFC+SDSS filter combinations at the wavelengths for several strong emission lines seen in Cas A ejecta. In addition, we tabulated the observed (uncorrected for reddening) relative fluxes seen for a typical S, O, Ar main shell knot (FMK2; Hurford \& Fesen 1996) and the resulting relative fluxes that would be detected using ACS/WFC with the SDSS filters. These values show that while the F625W filter is sensitive to emission from oxygen and sulfur (and nitrogen if present), the F775W and F850LP filters are mainly sensitive to oxygen and sulfur emission, respectively.

Measured outer knot fluxes in the three filters are tabulated in Table 2 for the 178 outer knots or knot complexes identified in previous studies, along with cross-referenced knot IDs. The complete knot table (but without knot cross-listings) is available in the online electronic version of the The Astrophysical Journal Supplement. In addition to filter fluxes, the knot catalog lists outer knot ID, its J2000 coordinates, position angle, radial distance from the remnant's center of expansion, an estimate of its proper motion based on ACS/WFC 2004.18 and 2004.93 images, and our assigned knot type. Both in Table 2 and in the complete catalog, outer knots are listed in the catalog in order of increasing position angle (P.A.) around the remnant (measured eastward from due north $\left[\right.$ P.A. $\left.=0^{\circ}\right]$ ).

Qualitative emission differences among outer ejecta knots can be seen through the construction of color composite images using the F625W (red), F775W (green), and F850LP (cyan) image frames. Such a color composite image was presented in Fesen et al. (2006a; see their Fig. 3). Many outer knots are only visible on one or two individual filter images, leading to strong color differences.

Figure 1 shows a mosaic of ACS/WFC images of Cas A constructed from co-adding the $\mathrm{F} 625 \mathrm{~W}$ and $\mathrm{F} 775 \mathrm{~W}$ images for all six pointings. Positions of the individual ejecta knots are indicated by open colored circles. Enlarged finding charts for the 15 lettered subregions (A-O) are presented in Figure Sets 2 and 3 which show the subregions both with and without knot identifying color circles and J2000 coordinates. (Only region A is shown in print; see the electronic edition of the Supplement for regions $\mathrm{B}$ through $\mathrm{O}$ ). Following the color coding of Fesen et al. (2006a), red circles show knots with strong [N II] $\lambda \lambda 6548,6583$ line emission, green circles knots with strong $\left[\mathrm{O}_{\text {II }}\right] \lambda \lambda 7319,7330$ line emissions, and cyan circles FMK-like knots that emit most strongly in the [S II] $\lambda \lambda 6716,6731$ lines.

Plots of filter flux ratios allow quantitative measures of emission differences for the various types of outer knots. Figure 4 shows the observed $\mathrm{F} 625 \mathrm{~W} /(\mathrm{F} 775 \mathrm{~W}+\mathrm{F} 850 \mathrm{LP})$ ratio versus the F775W/F850LP ratio for all 1825 outlying knots in the catalog. (A similar plot, along with a discussion of representative knot spectra, was shown in Fesen et al. [2006a], but only for 229 knots lying along the remnant's eastern limb.) The three types of outer knots can be seen to occupy different regions in this plot. Since FMK-like knots often show both strong [S II] and [O II] line emission, we used existing spectra of oxygen-rich knots, i.e., those showing a spectrum dominated by [O I] $\lambda \lambda 6300,6364$ and [O II] $\lambda \lambda 7319,7330$ line emission, to help distinguish these from knots which show strong lines of both [O I] and [S II], which are both detected in the F625W filter.

This led to a flux ratio of F775W/(F625W+F850LP) $\geq 1.0$ (not plotted) to be chosen to separate the [O II]-strong FMKs from the [S II]-strong FMKs; that is, those knots with stronger $[\mathrm{O}$ II $] \lambda \lambda 7319,7330$ emission detected via the $\mathrm{F} 775 \mathrm{~W}$ filter than the combined strength of [S III] and [S II] emissions detected in 
TABLE 2

Catalog of Outlying Ejecta Knots in Cas A

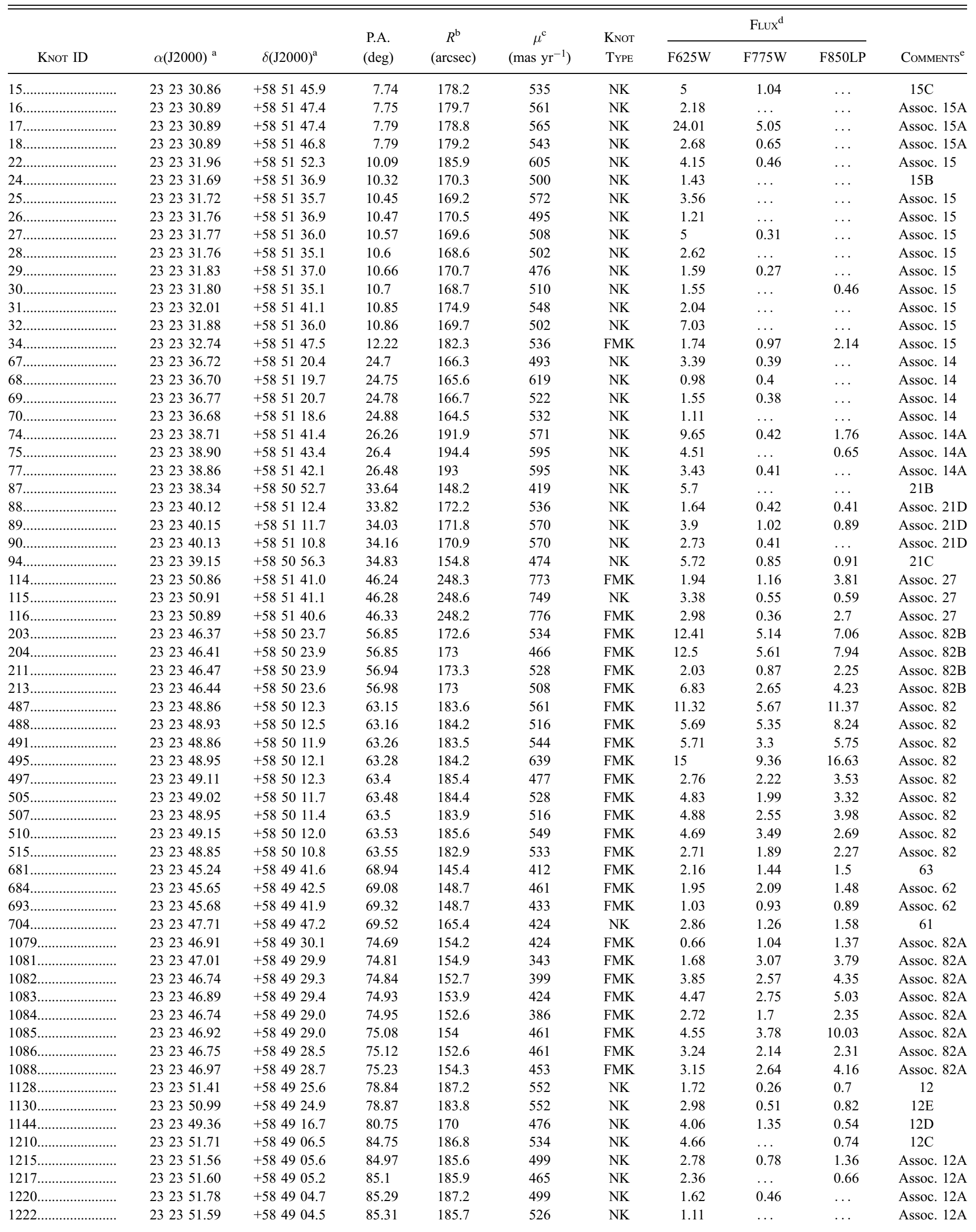


TABLE 2-Continued

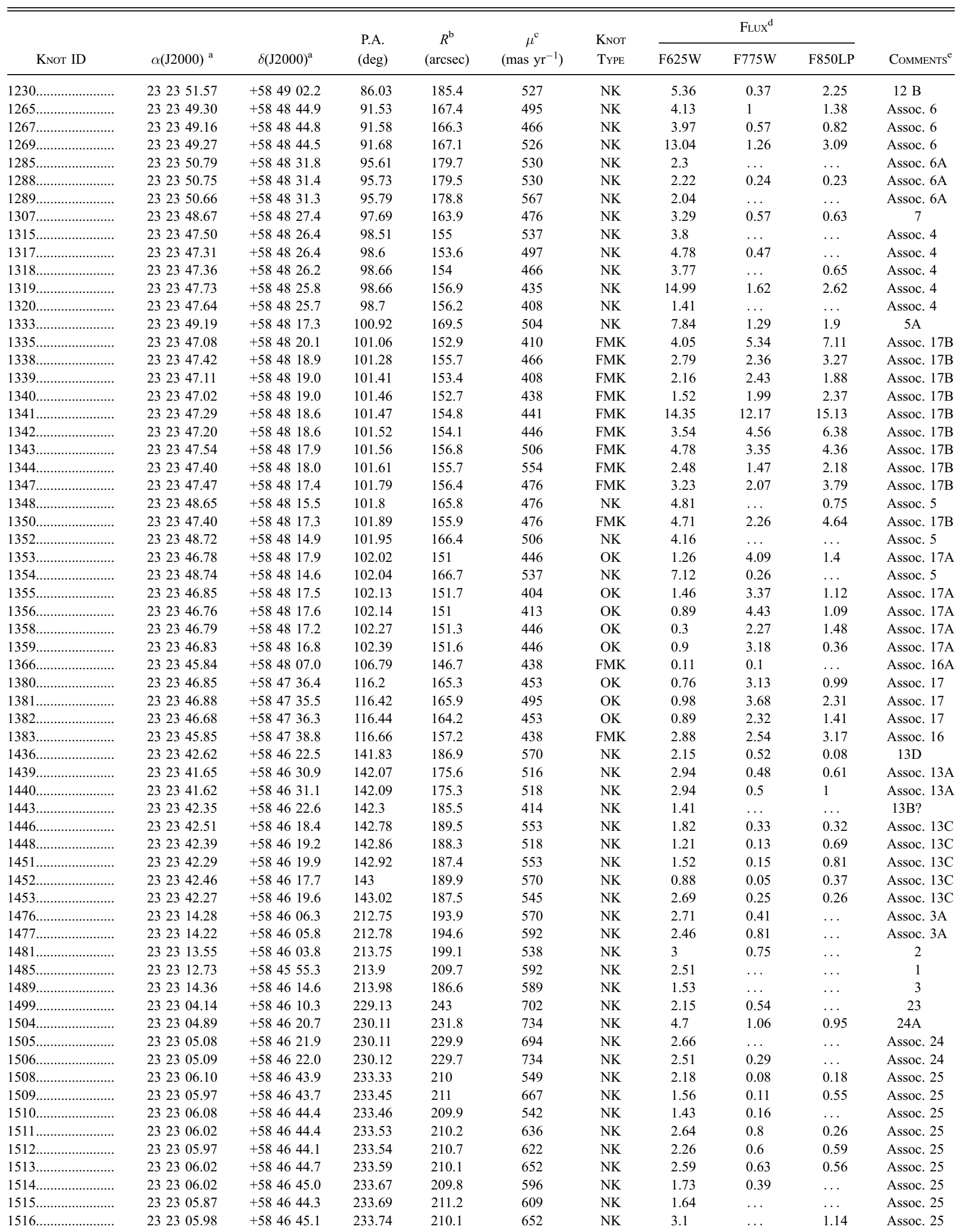


TABLE 2-Continued

\begin{tabular}{|c|c|c|c|c|c|c|c|c|c|c|}
\hline \multirow[b]{2}{*}{ KNOT ID } & \multirow[b]{2}{*}{$\alpha(\mathrm{J} 2000)^{\mathrm{a}}$} & \multirow[b]{2}{*}{$\delta(\mathrm{J} 2000)^{\mathrm{a}}$} & \multirow{2}{*}{$\begin{array}{l}\text { P.A. } \\
\text { (deg) }\end{array}$} & \multirow{2}{*}{$\begin{array}{c}R^{\mathrm{b}} \\
(\operatorname{arcsec})\end{array}$} & \multirow{2}{*}{$\begin{array}{c}\mu^{\mathrm{c}} \\
\left(\operatorname{mas}_{\mathrm{yr}} \mathrm{yr}^{-1}\right)\end{array}$} & \multirow{2}{*}{$\begin{array}{l}\text { KNOT } \\
\text { TYPE }\end{array}$} & \multicolumn{3}{|c|}{ FLux $^{d}$} & \multirow[b]{2}{*}{ Comments $^{\mathrm{e}}$} \\
\hline & & & & & & & F625W & F775W & F850LP & \\
\hline $1517 \ldots$ & 232305.94 & +584645.0 & 233.76 & 210.4 & 596 & NK & 3.78 & 0.17 & 0.66 & Assoc. 25 \\
\hline $1518 \ldots \ldots$ & 232306.00 & +584645.3 & 233.76 & 209.8 & 712 & NK & 1.92 & 0.5 & 0.19 & Assoc. 25 \\
\hline 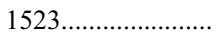 & 232306.17 & +584658.0 & 236.44 & 201.4 & 609 & NK & 6.34 & 1.61 & 1.07 & 26 \\
\hline 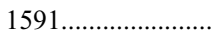 & 232302.48 & +584731.7 & 248.45 & 211.3 & 683 & FMK & 0.38 & 1.16 & 2.29 & $19 \mathrm{~F}$ \\
\hline $1622 \ldots$ & 232301.61 & +584822.5 & 262.47 & 205 & 318 & $\mathrm{OK}$ & $\ldots$ & 0.7 & $\ldots$ & Assoc. 19G \\
\hline $1623 \ldots$ & 232301.50 & +584822.4 & 262.49 & 205.8 & 600 & FMK & 0.5 & 0.61 & 2.49 & Assoc. 19G \\
\hline $1625 \ldots$ & 232301.75 & +584822.8 & 262.53 & 203.9 & 424 & FMK & 1.07 & 1.34 & 1.02 & Assoc. $19 \mathrm{G}$ \\
\hline $1632 \ldots \ldots \ldots \ldots \ldots \ldots$ & 232302.63 & +584827.2 & 263.53 & 196.6 & 502 & FMK & 1.15 & 0.46 & 2.61 & Assoc. 19E \\
\hline 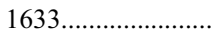 & 232302.68 & +584827.3 & 263.55 & 196.2 & 589 & FMK & 0.93 & 0.97 & 3.01 & Assoc. 19E \\
\hline $1643 \ldots \ldots$ & 232304.27 & +584833.8 & 265.14 & 183.2 & 496 & FMK & 1.96 & 8.97 & 21.63 & Assoc. $19 \mathrm{~K}$ \\
\hline 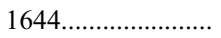 & 232304.43 & +584834.1 & 265.19 & 182 & 506 & FMK & 1.07 & 0.94 & 3.1 & Assoc. $19 \mathrm{~K}$ \\
\hline $1645 \ldots \ldots \ldots \ldots \ldots \ldots$ & 232304.21 & +584834.0 & 265.21 & 183.7 & 380 & FMK & 3.56 & 7.91 & 19.35 & Assoc. 19K \\
\hline 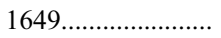 & 232304.28 & +584834.9 & 265.48 & 183.1 & 438 & FMK & 0.08 & 2.31 & 3.8 & Assoc. $19 \mathrm{~K}$ \\
\hline $1661 \ldots$. & 232303.10 & +584840.8 & 267.45 & 191.8 & 538 & FMK & 0.37 & 0.24 & 1.77 & 19D \\
\hline $1667 \ldots$ & 232303.33 & +584844.6 & 268.56 & 189.9 & 495 & FMK & 0.32 & $\ldots$ & 1.98 & $19 \mathrm{C}$ \\
\hline $1672 \ldots$ & 232303.18 & +584850.8 & 270.45 & 191 & 530 & FMK & 1.16 & 0.55 & 4.76 & 19B \\
\hline $1679 \ldots$. & 232301.56 & +584856.7 & 272.07 & 203.7 & 579 & FMK & 1.66 & 0.44 & 4.78 & Assoc. $19 \mathrm{H}$ \\
\hline $1680 \ldots \ldots \ldots \ldots \ldots \ldots \ldots \ldots \ldots \ldots \ldots$ & 232301.59 & +584856.8 & 272.1 & 203.5 & 619 & FMK & 1.01 & 0.74 & 2.12 & Assoc. $19 \mathrm{H}$ \\
\hline $1686 \ldots \ldots$ & 232302.96 & +584903.6 & 274.23 & 193.2 & 599 & FMK & 1.44 & 0.97 & 2.22 & $19 \mathrm{~A}$ \\
\hline $1693 \ldots \ldots$ & 232303.78 & +584905.2 & 274.86 & 187 & 629 & FMK & 5.84 & 6.08 & 5.91 & $19 \mathrm{~J}$ \\
\hline $1702 \ldots \ldots \ldots \ldots \ldots \ldots$ & 232303.06 & +584906.6 & 275.15 & 192.7 & 599 & NK & 3.56 & 1.44 & 1.62 & 19 \\
\hline 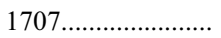 & 232303.95 & +584907.9 & 275.73 & 186 & 599 & FMK & 3.22 & 3.6 & 5.41 & $19 \mathrm{I}$ \\
\hline $1710 \ldots \ldots \ldots \ldots \ldots \ldots$ & 232305.59 & +584907.4 & 275.99 & 173.2 & 527 & OK & $\ldots$ & 1.57 & $\ldots$ & Assoc. 20 \\
\hline $1711 \ldots \ldots$ & 232305.51 & +584907.5 & 276.01 & 173.9 & 599 & FMK & 1.06 & 0.15 & 1.85 & Assoc. 20 \\
\hline $1719 \ldots \ldots$ & 232305.24 & +584933.1 & 284.05 & 180.4 & 559 & FMK & 2.58 & 0.99 & 4.56 & Assoc. 18 \\
\hline $1720 \ldots \ldots$ & 232305.52 & +584932.7 & 284.08 & 178.2 & 577 & FMK & 2.84 & 13.3 & 26.11 & Assoc. 18 \\
\hline 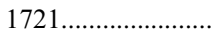 & 232305.54 & +584932.8 & 284.13 & 178 & 544 & FMK & 2.83 & 7.58 & 14.94 & Assoc. 18 \\
\hline $1722 \ldots$. & 232305.48 & +584933.1 & 284.2 & 178.5 & 514 & FMK & 1.5 & 2.86 & 7.52 & Assoc. 18 \\
\hline $1723 \ldots \ldots \ldots \ldots \ldots \ldots \ldots$ & 232305.53 & +584933.0 & 284.2 & 178.2 & 556 & FMK & 3.25 & 1.67 & 10.18 & Assoc. 18 \\
\hline 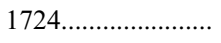 & 232305.61 & +584932.8 & 284.2 & 177.5 & 607 & FMK & 3.11 & 1.87 & 7.63 & Assoc. 18 \\
\hline $1725 \ldots \ldots \ldots \ldots \ldots \ldots \ldots$ & 232305.31 & +584933.5 & 284.21 & 179.9 & 534 & FMK & 5.55 & 3.89 & 12.03 & Assoc. 18 \\
\hline $1726 \ldots \ldots \ldots \ldots \ldots$ & 232305.66 & +584932.8 & 284.22 & 177.1 & 595 & FMK & 1.95 & 1.1 & 5.54 & Assoc. 18 \\
\hline $1727 \ldots$ & 232305.37 & +584933.5 & 284.26 & 179.5 & 595 & FMK & 3.54 & 3.52 & 15.2 & Assoc. 18 \\
\hline $1728 \ldots \ldots$ & 232305.55 & +584933.2 & 284.28 & 178.1 & 496 & FMK & 2.22 & 1.34 & 6.49 & Assoc. 18 \\
\hline $1729 \ldots \ldots \ldots \ldots \ldots \ldots \ldots$ & 232305.58 & +584933.5 & 284.39 & 177.9 & 552 & FMK & 2.31 & 1.04 & 5.61 & Assoc. 18 \\
\hline 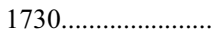 & 232305.41 & +584934.2 & 284.47 & 179.4 & 536 & $\mathrm{OK}$ & 0.53 & 1.54 & 0.88 & Assoc. 18 \\
\hline 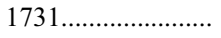 & 232305.68 & +584934.3 & 284.69 & 177.4 & 536 & FMK & 1.59 & 2.41 & 3.49 & Assoc. 18 \\
\hline $1732 \ldots \ldots \ldots \ldots \ldots \ldots$ & 232305.43 & +584934.9 & 284.72 & 179.4 & 552 & FMK & 3.82 & 4.58 & 7.98 & Assoc. 18 \\
\hline $1733 \ldots \ldots$ & 232305.49 & +584934.9 & 284.74 & 178.9 & 552 & FMK & 3.98 & 5.15 & 10.25 & Assoc. 18 \\
\hline 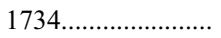 & 232305.60 & +584934.7 & 284.77 & 178.1 & 559 & FMK & 2.74 & 2.29 & 4.77 & Assoc. 18 \\
\hline $1735 \ldots \ldots \ldots \ldots \ldots \ldots$ & 232305.66 & +584934.6 & 284.77 & 177.5 & 572 & FMK & 2.45 & 2.9 & 4.39 & Assoc. 18 \\
\hline 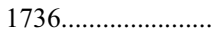 & 232305.69 & +584934.6 & 284.79 & 177.3 & 547 & FMK & 2.95 & 2.33 & 5.12 & Assoc. 18 \\
\hline $1737 \ldots \ldots \ldots \ldots \ldots$ & 232305.88 & +584934.4 & 284.85 & 175.9 & 520 & FMK & 1.29 & 4.3 & 6.79 & Assoc. 18 \\
\hline $1738 \ldots \ldots$ & 232305.92 & +584934.5 & 284.91 & 175.6 & 558 & FMK & $\ldots$ & 1.78 & 2.78 & Assoc. 18 \\
\hline 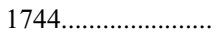 & 232308.49 & +585048.0 & 308.43 & 191 & 562 & NK & 9.44 & 0.8 & 1.16 & $9 \mathrm{~A}$ \\
\hline 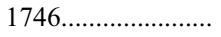 & 232308.64 & +585047.4 & 308.5 & 189.7 & 586 & NK & 6.42 & 1 & 3.03 & $9 \mathrm{~B}$ \\
\hline $1748 \ldots \ldots \ldots \ldots \ldots \ldots$ & 232307.69 & +585053.4 & 308.54 & 199.2 & 586 & NK & 16.92 & 1.8 & 2.95 & 10 \\
\hline 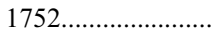 & 232313.74 & +58 5019.4 & 309.61 & 141.3 & 466 & FMK & 2.07 & 5.93 & 4.5 & Assoc. 10A \\
\hline 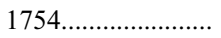 & 232308.66 & +585056.5 & 310.63 & 195.4 & 566 & NK & 5.45 & $\ldots$ & 0.51 & Assoc. $10 \mathrm{~A}$ \\
\hline 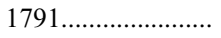 & 232311.72 & +585118.3 & 320.11 & 194.2 & 614 & $\mathrm{NK}$ & 6.05 & 0.38 & 1.17 & 11 \\
\hline $1802 \ldots \ldots \ldots \ldots \ldots \ldots$ & 232311.99 & +585126.1 & 322.02 & 198.9 & 576 & $\mathrm{NK}$ & 17.72 & 1.76 & 2.87 & 8 \\
\hline
\end{tabular}

NoтE.-Table 2 is available in its entirety in the electronic edition of the Astrophysical Journal Supplement. A portion is shown here for guidance regarding its form and content.

${ }^{\mathrm{a}}$ Knot positions were measured from ACS/WFC 2004.2 images. Units of right ascension are hours, minutes, and seconds, and units of declination are degrees, arcminutes, and arcseconds.

${ }^{b}$ Radial distances are measured with respect to the nominal center of expansion (Thorstensen et al. 2001) using the ACS/WFC 2004.2 images.

c Proper motions were estimated from ACS/WFC 2004.18 and 2004.93 images.

d Measured fluxes are in units of $10^{-17} \mathrm{erg} \mathrm{cm}^{-2} \mathrm{~s}^{-1}$.

e Corresponding knot identifications and associations as listed in Fesen (2001). 


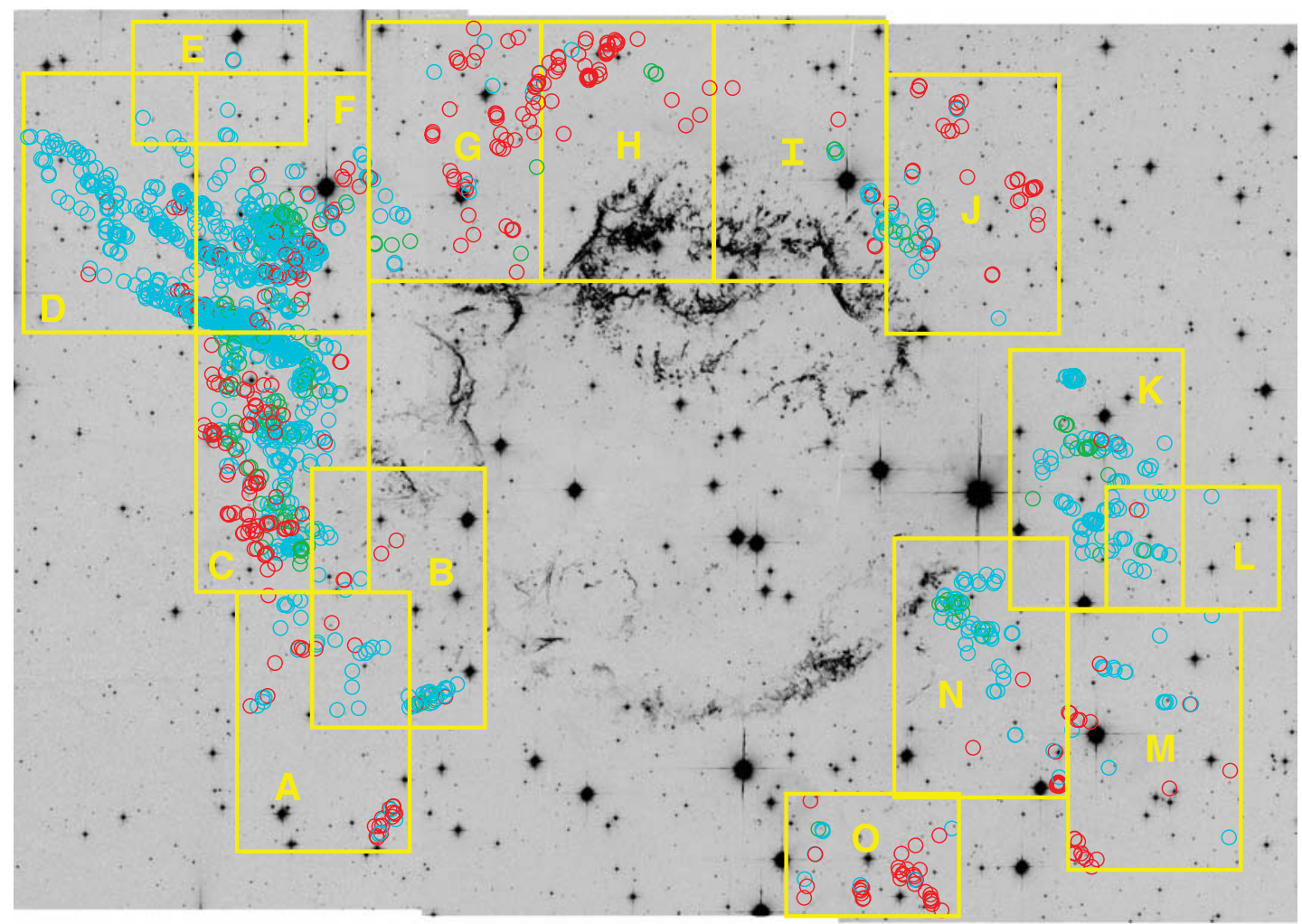

FIG. 1.- Mosaic of HST ACS images of Cas A taken in 2004 March. Image represents a mosaic of added F625W and F775W filter images for six ACS pointings ( NE, $\mathrm{SE}, \mathrm{N}$ central, S central, NW, and SW). Yellow rectangles mark the regions displayed in detail in subsequent finding chart figures, with the 15 regions (A through O) labeled alphabetically beginning in the southeast and progressing clockwise. Small open circles denote outer knot positions, with red circles marking strong [N II] $\lambda \lambda 6548,6583$ knots, green circles strong [O II] $\lambda \lambda 7319,7330$ knots, and cyan circles strong [S II] $\lambda \lambda 6716,6731$ FMK-like knots.

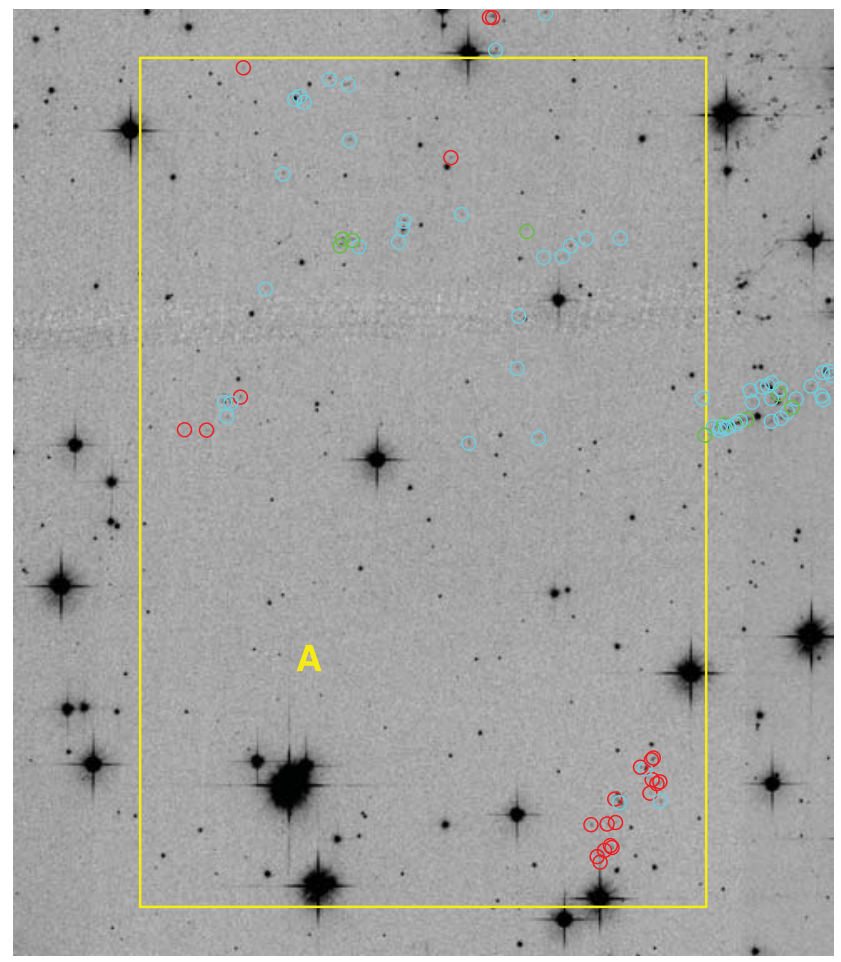

FIG. 2.-Finding chart for outlying ejecta knots in region A (see Fig. 1). Small open circles indicate outer ejecta knot positions, with red, green, and cyan circles marking strong [N II], [O II], or [S II] emission knots, respectively. [See the electronic edition of the Supplement for regions $B-O$.]

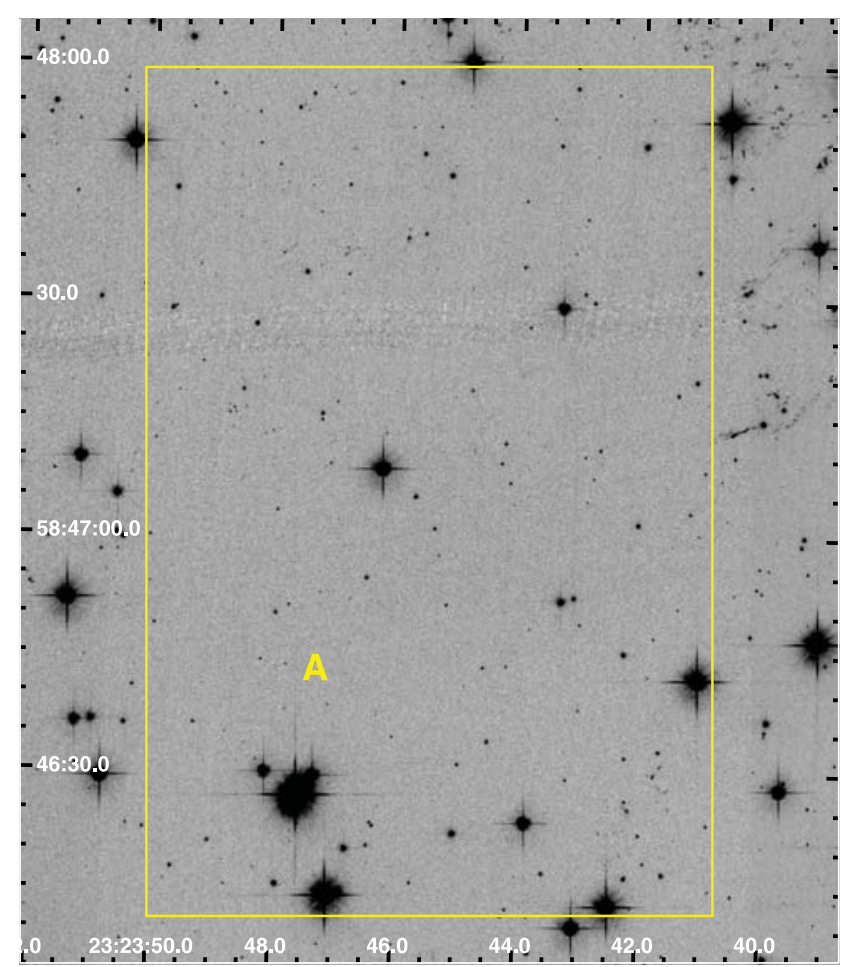

FIG. 3.-Same regions as in Fig. 2, but with knot ID circles removed and J2000 coordinates included. [See the electronic edition of the Supplement for regions $B-O$.] 


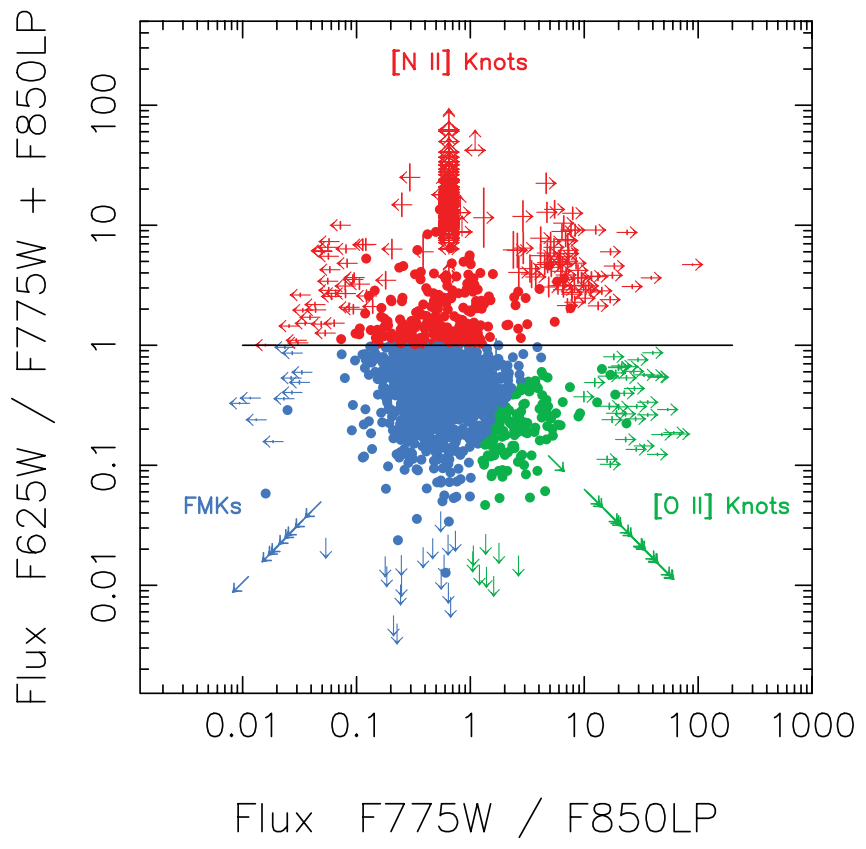

FIG. 4.-Plot of observed HSTACS F625W/(F775W + F850LP) vs. F775W/ F850LP flux ratios for all 1825 outer ejecta knots listed in the catalog. Knots are color-coded by emission type, with cyan indicating FMK-like knots, red indicating strong [ $\mathrm{N}$ II] emission knots, and green strong [O $\mathrm{O}$ ] emission knots. Arrows indicate a nondetection in at least one filter, with the vertical lines on the arrows indicating probable measurement error in the measured flux ratio.

the F850LP filter and the [O I] and [S II] emissions detected in the F625W filter. Consequently, knots with strong [O II] emission appear along the right-hand side of the plot and merge with the more common FMK knots at smaller F775W/F850LP and larger $\mathrm{F} 775 \mathrm{~W} /(\mathrm{F} 625 \mathrm{~W}+\mathrm{F} 850 \mathrm{LP})$ ratios.

Similarly, knots with strong [N II] $\lambda \lambda 6548,6583$ line emission were selected via $\mathrm{F} 625 \mathrm{~W} /(\mathrm{F} 775 \mathrm{~W}+\mathrm{F} 850 \mathrm{LP}) \geq 1.0$, thereby selecting those knots where the combined flux of [O I $\lambda \lambda 6300$, 6364, [S II] $\lambda \lambda 6716,6731$, and [N II] $\lambda \lambda 6548,6583$ emission was greater than the sum of F775W flux (due mostly to [O II] emission) and F850LP flux (due to [S III] and [S II] emissions). Since both the observed [O I] flux rarely if ever exceeds the [O II] $\lambda \lambda 7319,7330$ flux, and the observed [S II] $\lambda \lambda 6716,6731$ emission is unlikely to ever exceed the combine flux of $[\mathrm{S}$ III] $\lambda \lambda 9069$, 9531 and [S II] $\lambda \lambda 10287-10370$ line emission (Hurford \& Fesen 1996; Winkler et al. 1991), then any knot for which the F625W/ ( $\mathrm{F} 775 \mathrm{~W}+\mathrm{F} 850 \mathrm{LP})$ ratio is $\geq 1.0$ requires the presence of significant $\left[\mathrm{N}_{\mathrm{II}}\right] \lambda \lambda 6548,6583$ emission. The value for the $\mathrm{F} 625 \mathrm{~W} /$ $(\mathrm{F} 775 \mathrm{~W}+\mathrm{F} 850 \mathrm{LP})$ ratio of $\geq 1.0$ was chosen based on spectral properties of a few dozen [ $\bar{N}$ II] emission-dominated knots, as well as ones that show "mixed" spectral emission properties, i.e., with both strong [ $\left.\mathrm{N}_{\mathrm{II}}\right]$ emission and FMK-like oxygen and sulfur line emission (Fesen 2001).

Of the 1825 knots identified and cataloged, the majority (1189) show flux ratios similar to those exhibited by main shell FMKs. These are color-coded cyan in Figure 4. Most of these lie in the $\mathrm{NE}$ and SW jet regions. An additional 444 knots have been classified as [N II] knots (red), with an additional 192 knots identified as showing strong [O II] emission knots (green), based mainly on their F775W/F850LP flux ratio.

We note that a number of the [N II] knots did not have sufficient F775W or F850LP flux to accurately plot their position on the diagram. Such knots often only show [N II] $\lambda \lambda 6548,6583$ line emission, with no measurable $[\mathrm{O}$ II] $\lambda \lambda 7319,7330$ and [S III]

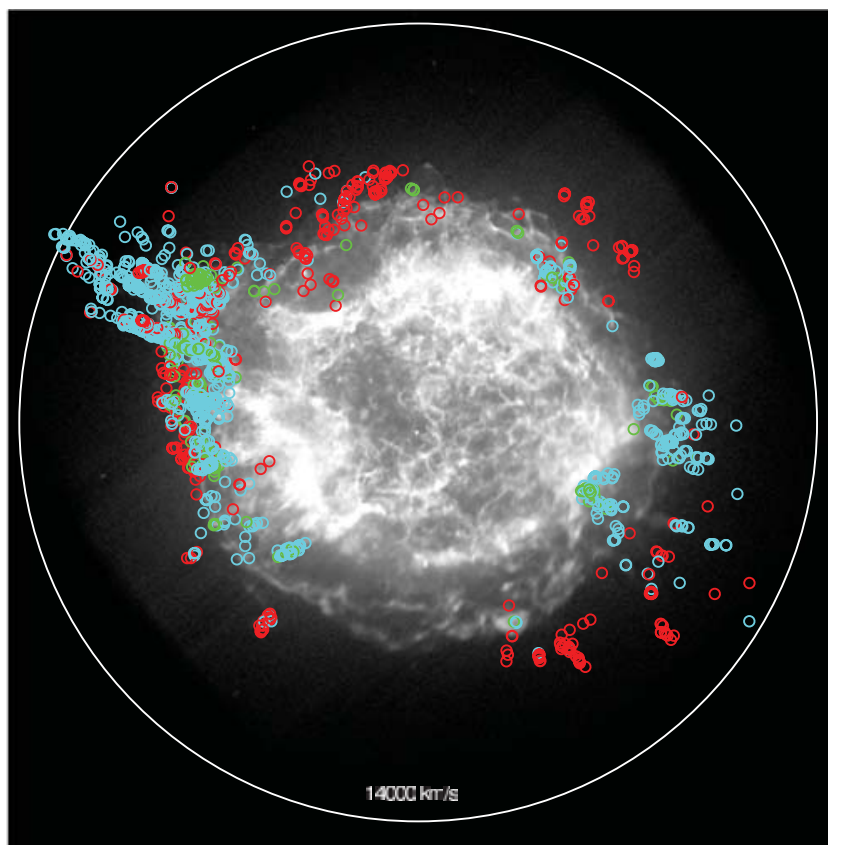

FIG. 5.- The 1 Ms exposure Chandra image of Cas A, with the locations of all 1825 identified outer ejecta knots marked, color coded by their emission properties. Red open circles indicate knots with strong [N II] line emission, green open circles knots with strong [O II] emission, and cyan open circles FMK-like outlying knots.

$\lambda \lambda 9069,9531$ detected in the F775W or F850LP filters. Consequently, this created a build-up of knots plotted with an apparent F775W/F850LP flux ratio around 0.7 due simply to the background flux differences in these filters.

\subsection{The Global Distribution of Outer Debris}

Our catalog of high-velocity outlying optical ejecta contains knots at projected radial distances of $104^{\prime \prime}$ to $299^{\prime \prime}$ from the center of expansion (Thorstensen et al. 2001). This places them close to or outside the remnant's $\simeq 6500 \mathrm{~km} \mathrm{~s}^{-1}$ forward shock front, as judged by the remnant's outermost X-ray emission (Gotthelf et al. 2001; DeLaney \& Rudnick 2003).

Figure 5 shows the plotted locations and overall distribution of these knots with respect to the remnant's X-ray emission as seen in the 1 Ms Chandra ACIS image (epoch 2004.3; Hwang et al. 2004). Debris knot positions are marked with open circles, color coded either red, green, or cyan to indicate those knots with spectra dominated by strong [N $\mathrm{N}$ II $\lambda \lambda 6548,6583$, [O II] $\lambda \lambda 7319$, 7330 , or $[\mathrm{S}$ II] $\lambda \lambda 6716,6731$ line emission, respectively.

As shown in Figure 5, the distribution of outer ejecta knots displays a strongly asymmetrical structure, due principally to the $\mathrm{NE}$ and SW jets, which appear as distinct and roughly opposing features, with the NE jet at P.A. $=55^{\circ}-75^{\circ}$, and the SW jet at P.A. $=230^{\circ}-280^{\circ}$. The large white circle in Figure 5 has a radius of $282^{\prime \prime}$ and marks the distance a knot would have reached having a constant proper motion of $0.868^{\prime \prime}$ for $325 \mathrm{yr}$, which translates to a transverse velocity of $14,000 \mathrm{~km} \mathrm{~s}^{-1}$ at Cas A's assumed distance of $3.4 \mathrm{kpc}$.

No outer knot of any type could be identified in two broad, opposing regions along the NW and SE sections of the remnant. These regions are centered near position angles $170^{\circ}$ and $342^{\circ}$. These apparent outer ejecta knot "gaps" are visible in both Figures 5 and 6 . While the significance of these northern and southern gaps is uncertain, the remnant's X-ray point source (Tananbaum 

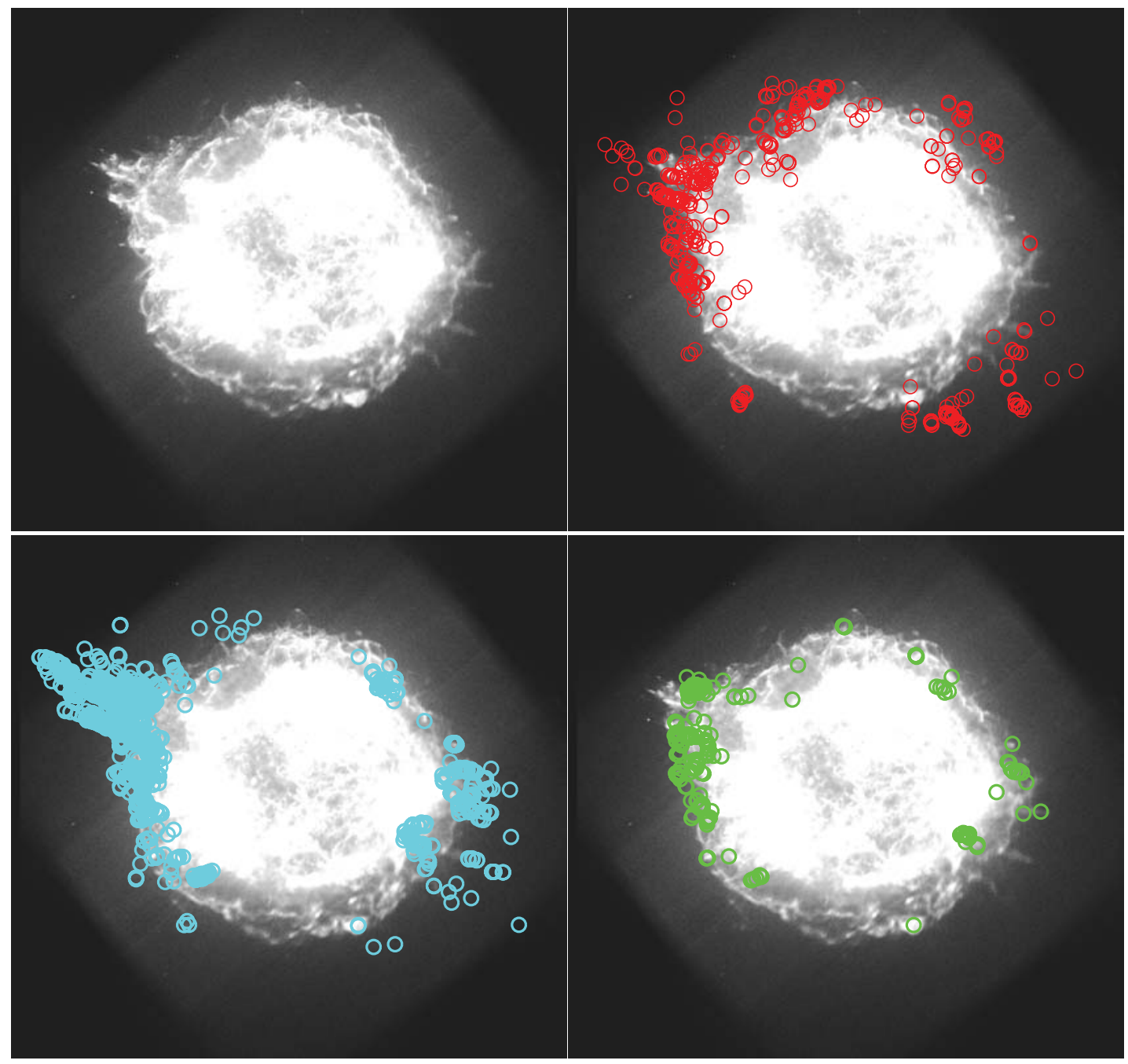

Fig. 6.-Top left: 1 Ms exposure Chandra image of Cas A, showing the maximum extent of the remnant's X-ray emission. The other three panels show the locations of outer optical knots on the remnant's X-ray emission, color coded by emission type (red: [N II] knots; green: [O II] knots; cyan: FMK-like knots).

1999) has an apparent proper motion of some $350 \mathrm{~km} \mathrm{~s}^{-1}$ in the direction of P.A. $=169^{\circ} \pm 8.4^{\circ}$, that is, toward the middle of the southern gap (see discussion in Fesen et al. 2006b).

As can also be seen qualitatively from Figure 5 and quantitatively from the values tabulated in our complete online knot cata$\log$, both the NE and SW jets of ejecta exhibit similar maximum radial distances and proper-motion-derived expansion velocities, namely, $r=290^{\prime \prime}-300^{\prime \prime}$ and $\mu=0.80-0.85$, implying $v_{\max } \simeq$ $14,000 \mathrm{~km} \mathrm{~s}^{-1}$. Thus, some jet knots reach out nearly to the white circle, and in a few cases just beyond.

Not only do the NE and SW jets contain the remnant's highest velocity optically emitting ejecta, but these knots tend to be FMK-like ejecta. This is in contrast to other regions where the nitrogen-rich ejecta (open red circles) represent the remnant's highest velocity debris $\left(10,000-11,000 \mathrm{~km} \mathrm{~s}^{-1}\right)$, followed by the O-rich ejecta, and then the S-rich, FMK-like knots (Fesen et al. 2006a).

In Figure 6, we show the $1 \mathrm{Ms}$ Chandra ACIS image (top left), with the locations of the three main types of outer ejecta optical knots plotted separately in the three adjacent panels. While the $\mathrm{NE}-\mathrm{SW}$ jet/counterjet asymmetry in the distribution of the remnant's outer optical ejecta knots can be seen most clearly in the distribution of the cyan FMK-like knots, there is some indication of it in the red [N II] knots (top right).

\subsection{Knot Types by Remnant Region}

We now examine the properties of outer ejecta knots divided into three regions: the NE jet, the SW jet, and all other "non-jet" areas. The exact divisions for these three regions are shown in Figure 7. We chose $\mathrm{PA}=55^{\circ}-77^{\circ}$ and $233^{\circ}-279^{\circ}$ for the NE and SW jet, respectively. Although the SW jet appears to cover a wider angular region than the NE jet, the exact regions chosen for the jets are somewhat subjective, and the precise position angle ranges selected here may not be physically meaningful.

In terms of the number of knots in these three periphery regions, we found 972, 207, and 646 knots in NE jet, SW jet, and non-jet regions, respectively, for a total of 1825 knots. Histogram plots for the three types of knots as a function of radial distance from Cas A's expansion center for the three remnant regions are shown in Figures 8-10, with Figure 11 showing the sum for all regions broken down by knot type.

\subsubsection{The NE and SW Jets}

In Figures 8 and 9, we show number histograms for the NE and SW jet regions divided by ejecta knot type as a function of radial distance from Cas A's center of expansion. These plots reveal that FMK-type ejecta knots greatly outnumber the other two types of knots in both jets. For example in the NE jet, FMK-like 


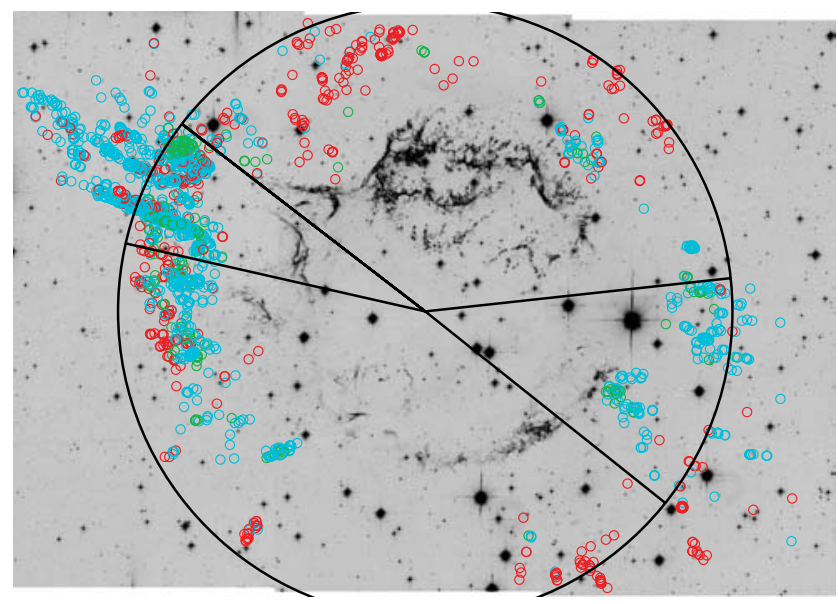

FIG. 7.- Mosaic of combined ACS/WFC F625W and F775W images of Cas A taken in 2004 March. Figure shows the specific regions chosen to represent the NE jet and SW jet regions. Outer circle shown marks a $200^{\prime \prime}$ radial distance from the center of expansion, corresponding to a measured ejecta knot transverse velocity of $\sim 10,000 \mathrm{~km} \mathrm{~s}^{-1}$ for an age of $320 \mathrm{yr}$.

knots outnumber [ $\mathrm{N}$ II] and [O II] type knots by factors of nearly 5 and 10 , respectively.

FMK-like knots also tend to be the most common type of ejecta at the greatest radial distances in both jets. The relative fractions of [ $\mathrm{N}$ II] knots to FMK-like knots, however, does not significantly change with increasing distance. That is, although far fewer in number, [ $\mathrm{N}$ II] type knots can be seen at the outer extents of both jets alongside the most distant FMK-like knots.

These distributions are in contrast to that seen for the [O II] type knots, which are fewer in number in both jets and are found exclusively at radial distances between $130^{\prime \prime}$ and $210^{\prime \prime}$. Also, unlike [N II] and FMK-like knots, [O II] knots in the two jets show a distribution that cuts off sharply at around $210^{\prime \prime}$. Despite this, for the NE jet where we have a sizable number of [N II] and [O II] type knots for comparison, the peaks in the radial distance distri-

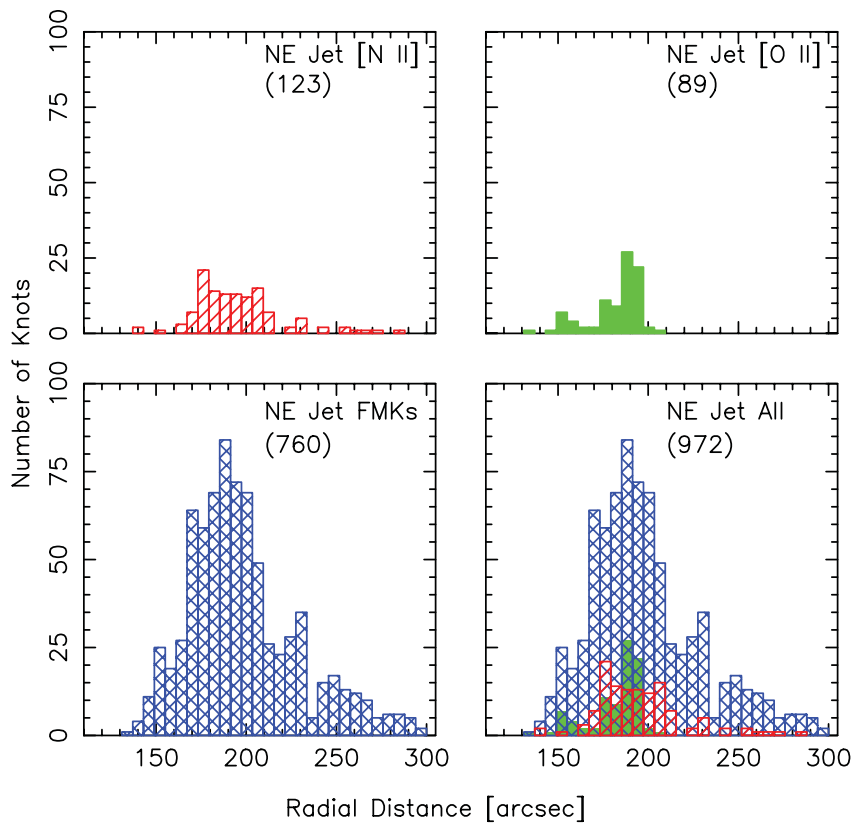

FIG. 8.-Histograms for the three emission classes of outer knots, for the NE jet region only.

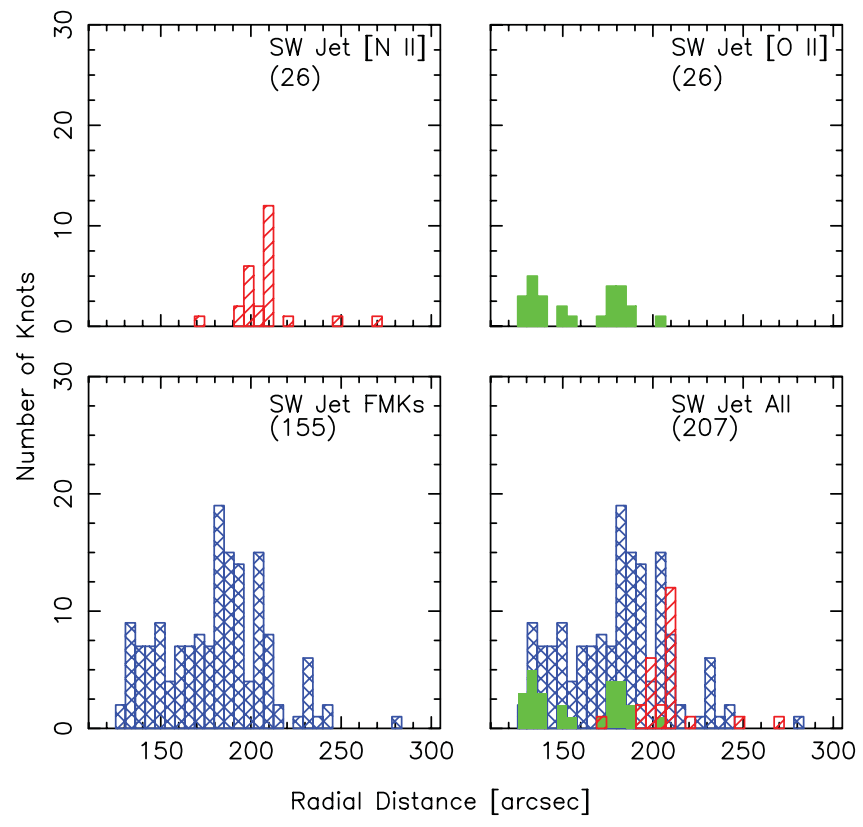

FIG. 9.- Histograms for the three emission classes of outer knots, for the SW jet region only.

bution for the different knot types are fairly similar (see Fig. 8, bottom right).

\subsubsection{Non-Jet Regions}

In non-jet regions, the situation appears somewhat different. As shown in Figure 10, [N II] type knots are seen to extend the farthest out, to nearly $250^{\prime \prime}$ from the expansion center, with a distribution peak at a greater distance $\left(180^{\prime \prime}\right)$ than FMK-like knots $\left(160^{\prime \prime}\right)$. In terms of projected radial distances, [O II] type knots are more concentrated, with a distribution peak at $160^{\prime \prime}$. The generally larger distances and broader range of distances for non-jet $[\mathrm{N}$ II] knots compared to the other two types of ejecta is shown in
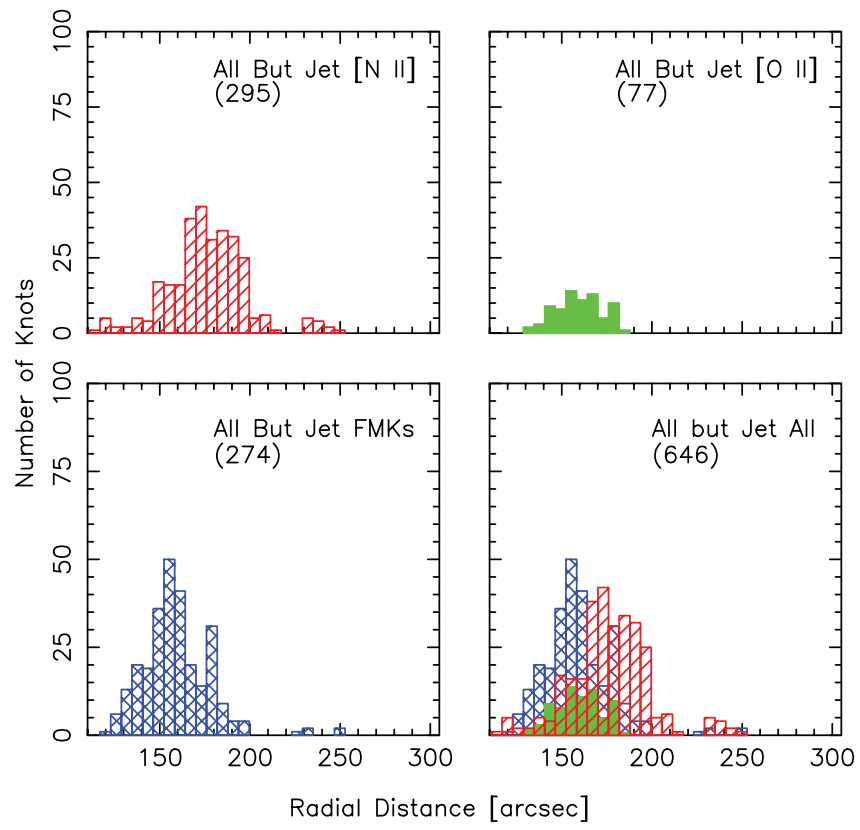

FIG. 10.- Histograms for the three emission classes of outer knots in all except for the NE and SW jet regions. 


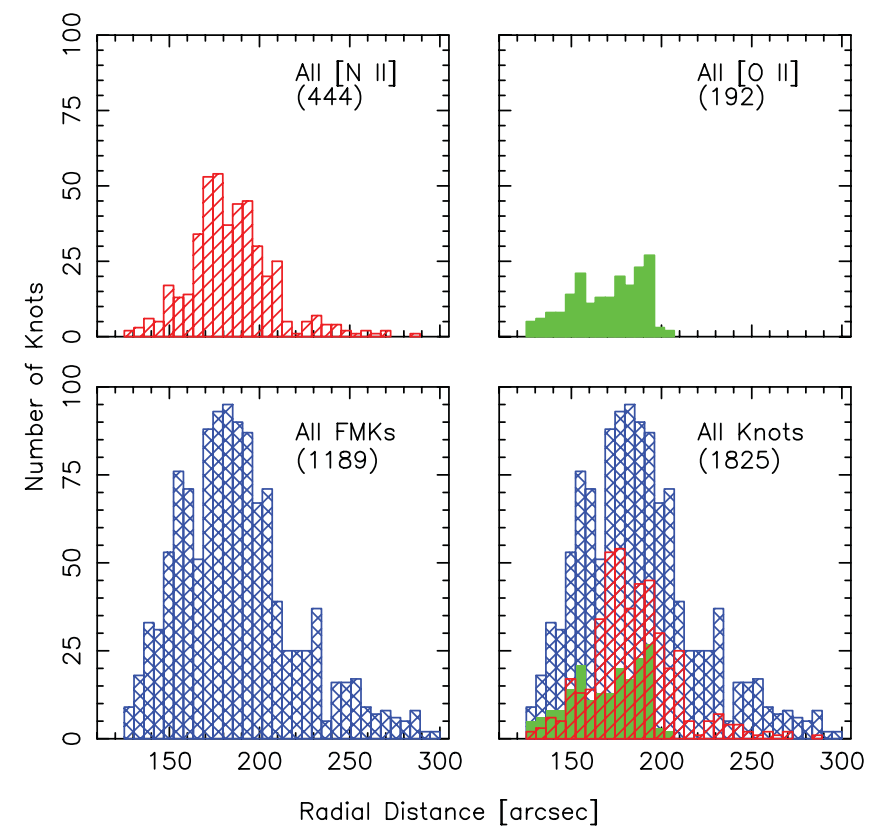

Fig. 11.- Histograms for the three emission classes of outer knots in all regions.

the bottom right panel of Figure 10, where all three knot types are plotted. This shows that for radial distance above 180", [N II] type knots are the most common.

Considering all radial distances, [N II] type knots are the most common type of outer debris in non-jet regions, accounting for about $45 \%$ of these knots, which is just slightly greater than seen for FMK-like knots, but almost 4 times that for [O II] knots. If one excludes the eastern limb of the remnant, where all three types of knots are relatively numerous, [N $\mathrm{N}$ ] type knots then become by far the most common type of knot detected (see Figs. 5 and 6).

\subsubsection{Summary for All Regions}

Figure 11 shows histograms for all 1825 outer knots found in all regions plotted as a function of radial distance from Cas A's center of expansion. The dramatic cutoff at $r \sim 200^{\prime \prime}$ for [O II] type knots is readily apparent, as is the dominance of FMK-like knots in the overall population of the remnant's outermost ejecta. This is not surprising, given that the most visible outer ejecta knots, namely those on the NE jet, number some 850 knots (knot IDs from around 100 to 950), the majority of which are FMKlike knots.

In addition, Figure 11 shows that although [N II] type knots can be seen out to $280^{\prime \prime}$, there appears to be a steep decline in the number of $[\mathrm{N} \mathrm{II}]$ knots at radial distances beyond $210^{\prime \prime}$. This cutoff is mainly due to knots outside of the jet region, which contain nearly two-thirds of all [ $\mathrm{N}$ II] knots detected around the remnant. Therefore, while there is little difference in the distribution peaks between [N II] and FMK-like knots, the drop-off of [N II] knots at large distances contributes to a distribution profile different from that of FMK-like knots, with the [N II] knots showing a much flatter distribution curve beyond $210^{\prime \prime}$.

\subsection{Outer Knot Proper Motions}

Proper motion estimates are listed for all 1825 knots in the complete online catalog, and for just those outer knots previously identified and studied in the print Table 2. Not surprisingly, in view of their generally greater radial distance, Cas A's outer

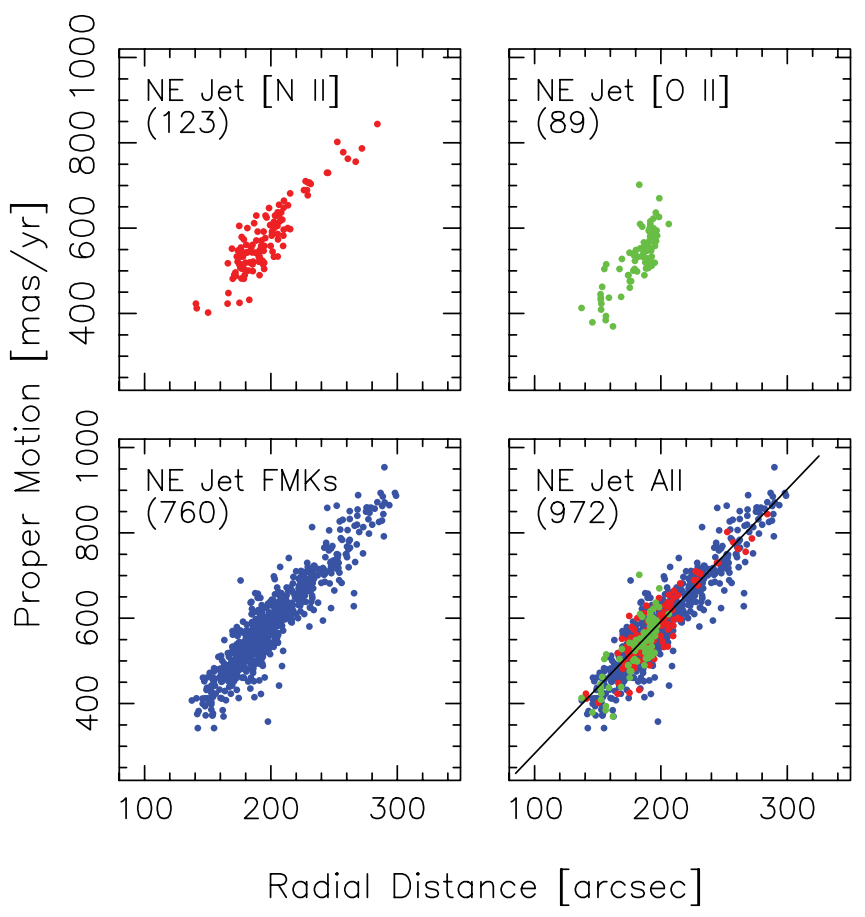

FIG. 12.-Measured proper motions as a function of radial distance from the expansion center for outer knots in NE jet region only.

knots exhibit relatively large proper motions compared to main shell ejecta filaments and knots, with values consistent with the observed projection position from the remnant expansion center.

The majority of outer knots in the catalog have measured proper motion between $0.30^{\prime \prime}$ and $0.90^{\prime \prime}$. At a distance of $3.4 \mathrm{kpc}$, these values imply projected transverse velocities of about 4800 to $14,500 \mathrm{~km} \mathrm{~s}^{-1}$. We estimated proper motion measurement errors to range from $0.015^{\prime \prime}(1 \sigma)$ for the brightest $[F(\lambda)>$ $\left.10^{-16} \mathrm{erg} \mathrm{cm}^{-2} \mathrm{~s}^{-1}\right]$ and pointlike (spherical shape and/or unresolved in the WFC images) knots to $0.025^{\prime \prime}$ for faint and/or highly asymmetrically shaped knots.

A comparison of our proper-motion estimates with those of Fesen (2001) shows generally good agreement. For example, for the $\left[\mathrm{N}_{\mathrm{II}}\right]$ type knots seen along the eastern limb of the remnant, our values and those of Fesen (2001) (listed here in brackets) for knots 15, 15A, 15B, 15C, 21A, 21B, 21C, and 21D are (in arcsec): 0.515 [0.53], 0.546 [0.56], 0.572 [0.58], 0.561 [0.58], 0.411 [0.42], 0.472 [0.49], and 0.536 [0.54], respectively.

One significant difference between these two values, however, is that ours are based on actual measured knot motions over a 9 month period, whereas those of Fesen (2001) were estimates of inferred proper motions assuming an explosion date of 1680 and a somewhat different explosion center, $\alpha(\mathrm{J} 2000)=23^{\mathrm{h}} 23^{\mathrm{m}} 23.70^{\mathrm{s}}$, $\delta=58^{\circ} 48^{\prime} 47.4^{\prime \prime}$, compared to the Thorstensen et al. (2001) value we used of $\left.\alpha(\mathrm{J} 2000)=23^{\mathrm{h}} 23^{\mathrm{m}} 23.77^{\mathrm{s}}, \delta=58^{\circ} 48^{\prime} 49.4^{\prime \prime}\right)$. In addition, the earlier proper-motion estimates were based on groundbased images, where several closely spaced knots were often interpreted as a single feature.

Our estimated proper motions for the NE jet, SW jet, and all non-jet regions based on our ACS/WFC 2004.18 and 2004.93 image data are shown plotted separately in Figures $12-14$ relative to knot projected distances. The bottom right panels of these figures show the three knot types overplotted together. The straight line corresponds to a constant rate of expansion assuming an explosion date of 1671, as estimated by Thorstensen et al. (2001). 


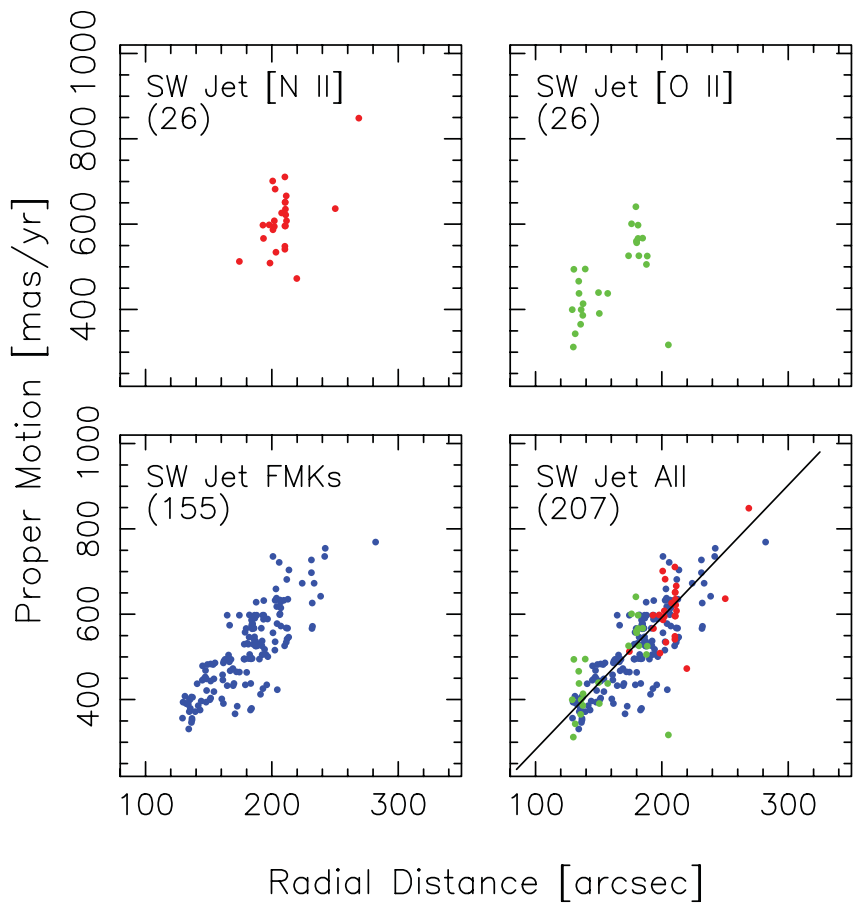

FIG. 13.-Measured proper motions as a function of radial distance from the expansion center for outer knots in the SW jet region only.

This line fits the measured proper motion as a function of radial distance well, suggesting no significant difference in the deceleration (or acceleration) with type of ejecta knot or knot distance.

As shown in the summary plots of Figure 15, the line of constant expansion for an age of $333 \mathrm{yr}(2004-1671)$ passes through the middle of all three ejecta types, right out to their farthest extent. A handful of outliers are seen below this line, which could indicate either misidentified main shell fragments, which are known



FIG. 14.-Measured proper motions as a function of radial distance from the expansion center for outer knots in all except for the NE and SW jet regions.

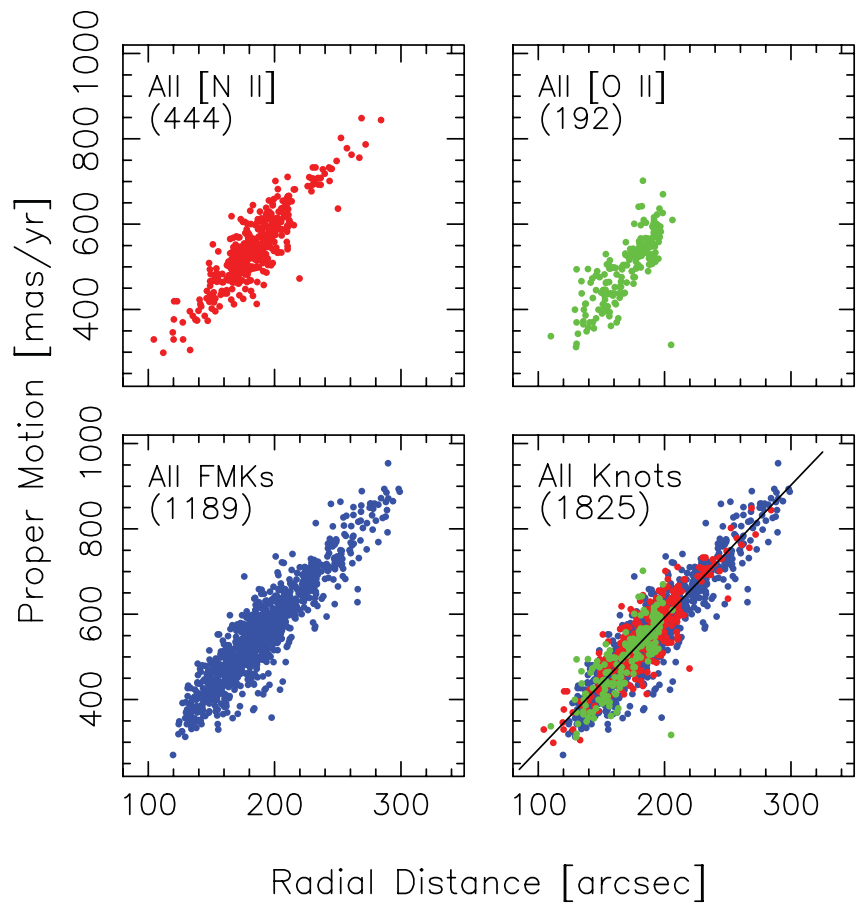

FIG. 15.-Measured proper motions as a function of radial distance from the expansion center for all outer knots.

to expand much slower, or simply poorly measured proper motions due to faintness on the ACS images and/or the relatively short 9 month time span between measured positions.

Figure 15 also highlights the cutoff of the [O II] at $200^{\prime \prime}$, as well as a sharp drop-off in the number of [ $\mathrm{N}$ II] beyond $220^{\prime \prime}$. The [O II] type knots show the smallest position and proper motion range, falling in between the FMK and [N II] type knots for both position and proper motion. Along the remnant's eastern limb, Fesen et al. (2006a) translated the measured proper motions of 69 [N II], 40 [O II], and 120 FMK-like knots into expansion velocities of 8100,7900 , and $7600 \mathrm{~km} \mathrm{~s}^{-1}$, respectively. They attributed this pattern to the progenitor's N, He-O-S, Ar, Si-rich layers remaining intact after supernova explosion and expansion.

\section{DISCUSSION}

Our catalog of outer ejecta in Cas A using HST imaging presents a more complete census of Cas A's highest velocity debris than previously reported. These data, however, do not push the limits of what outlying ejecta can be detected. Although we have cataloged nearly 2000 outlying knots, there could be hundreds more around the periphery of the remnant, and longer exposure HST images taken with narrower filters like those available using WFPC3 are likely to reveal many more outer knots. Detecting a significant number of additional outlying ejecta would give us a more complete and hence a more accurate picture of the distribution of this remnant's fastest ejecta.

At present, Cas A is the only young remnant to exhibit such small high-velocity outlying ejecta. Studies of such high-speed ejecta can give useful insights into the chemical and dynamical properties of the progenitor's outermost layers and the dynamics of the SN explosion. In addition, this catalog of knots should prove to be a valuable tool for studies investigating high-velocity knot flux variability due to passage through the surrounding circumstellar and interstellar medium, along with better measurements of proper motion, possible knot deceleration, mass ablation, and knot disruption. 




FIG. 16. - Combined ACS F625W and F775W images for Cas A's northeast jet (left) and southwest jet (right) regions, showing tight clusters of [O II] emission knots (yellow open circles) along the edges of the jet FMK-like knots (white open circles).

In light of the previously reported results from this survey, which discussed the discovery of outlying high-velocity oxygenrich ejecta and the remnant's expansion asymmetry (Fesen et al. 2006a, 2006b), we will limit our discussion here to just a few topics.

\subsection{Oxygen Knot Clusters at Jet Bases}

The distribution of outlying [O II] bright knots (green circles) appears to be the most limited of the three types of outer ejecta in terms of both position angle and radial distance out from the remnant center (see Fig. 6, bottom right). Interestingly, this type of outlying ejecta knot appears especially concentrated along the north and south edges of the NE jet, and possibly the SW jet as well. This is illustrated in Figure 16 where we plot strong [O II] emission knots on positive composite ACS/WFC F625W+F775W images of the NE and SW jet regions (left and right panels, respectively). For greater clarity, the $[\mathrm{O} \mathrm{II}]$ emission knots are color-coded yellow here instead of green, as elsewhere in this paper.

Along the northern edge of the NE jet's base, these presumably oxygen-rich knots form a relatively tight cluster of more than two dozen knots, with several other knots seen grouped along the jet's projected southern edge. The group of [O II] emission knots near the base of the jet's northern edge is an unusually rich cluster of such knots, not seen anywhere else in the remnant. Just south of this cluster, one finds only a few [O II] emission knots until reaching the jet's southern filament of FMK-like knots, where once again one sees numerous [O II] type knots. While far fewer $\left[\mathrm{O}_{\mathrm{II}}\right]$ knots are detected near the area of the $\mathrm{SW}$ jet, there are two tight clusters of this type of knot along the northern edge and near the southern section of the SW jet.

Few of these jet [O II] cluster knots had been previously reported for either jet region. They seemingly escaped easy detection due the combination of their faintness and their relative weakness in [O I] $\lambda 6300$ line emission, which would have allowed their detection in the F675W filter on the WFPC2 aboard HST (see Fesen et al. 2006a for a representative spectrum).

The nature and meaning of such O-rich clumps of material on either edge of the NE jet (and possibly the SW jet) is unclear. It might be build-up of material from the O-rich layer of the progenitor which was pushed aside by a violent ejection of under- lying material from a high-velocity jet from the progenitor's core region. However, explosive jet models like that of Mazzali et al. (2005) show that ejecta rich in iron, not oxygen, should line the outer edges of a jet. This brings us to the basic question of the true nature of these so-called jets of ejecta in the Cas A remnant.

\subsection{The Nature of the $N E$ and $S W$ "Jets"}

Observations of core-collapse $\mathrm{SNe}$, such as Cas A is believed to be, show high late-time linear polarization levels, suggesting that the innermost layers driving the $\mathrm{SN}$ expansion are asymmetrical (Wang et al. 1996, 2001; Leonard et al. 2000, 2001; Leonard \& Filippenko 2001). This has led some modelers to investigate the effects of rapid rotation and magnetic fields leading to magnetorotational jet models (Khokhlov et al. 1999; Höflich et al. 1999, 2001; Akiyama et al. 2003). Others have investigated asymmetric neutrino-driven models in order to generate aspherical, sometimes even jetlike, SN explosions (Burrows et al. 1995, 2005; Kifonidis et al. 2003, 2006; Yamasaki \& Yamada 2005; Wilson et al. 2005).

The overall asymmetrical appearance of Cas A's outer ejecta seen in Figure 5 is not unlike that seen in some asymmetric models of jet-induced explosion (Khokhlov et al. 1999; Mazzali et al. 2005; Wheeler et al. 2008). However, the true nature of Cas A's "jets" is currently controversial. From the little spectroscopic data that exists for the more prominent NE jet, it appears to be a fairly broad plume of ejecta with an opening angle of around $30^{\circ}$ (Fesen \& Gunderson 1996). Material located at the farthest tip of this jet, and thus having the highest ejection velocities, appears to be mainly sulfur-rich debris, with no significant line emission from oxygen. The few dozen or so NE and SW knots with radial velocity measurements seem to indicate that both jets lie fairly close to the plane of the sky, suggesting either a very fortuitous viewing angle or a more complex asymmetric explosion structure than a simple bipolar symmetry.

Despite their rough alignment on either side of the remnant, the data in hand do not establish that the NE and SW jets really comprise a coherent jet-counterjet expansion structure. Moreover, some recent observations have indicated that Cas A's ejecta structure may be much more complex than a simple bipolar expansion. Recent Spitzer data suggest a bipolar structure roughly perpendicular to the jet-counterjet axis, composed of fairly slow 
moving ejecta (Ennis et al. 2006), and Wheeler et al. (2008) argue that this new axis may represent the true jet line, with the apparent $\mathrm{NE}$ and $\mathrm{SW}$ jets created by nonaxisymmetric flows approximately along the progenitor's equatorial plane.

There are other means of producing an asymmetric debris structure besides a bipolar jet scenario. For example, in the Blondin et al. (1996) model for an axisymmetric circumstellar interaction, a jetlike feature of SN ejecta can be generated in the progenitor's equatorial plane by even mild pole/equator density gradients due to asymmetric mass loss during the red giant phase.

At present, is not clear if any of these jet or non-jet models can reproduce the expansion dispersion and the near constant rate of expansion for the various types of ejecta, such as that seen in Figure 15, or the apparent clumping of O-rich ejecta along the sides of the NE jet. Obviously, what is needed is a complete threedimensional kinematic and chemical mapping of Cas A's optical emission, including both NE and SW jets. Such a data set would give us a better understanding of how slow and fast ejecta in this core-collapse supernova remnant are arranged on large $(\sim 1 \mathrm{pc})$ and small ( $\sim 0.01 \mathrm{pc})$ spatial scales. When combined with Chandra and Spitzer velocity and abundance data (e.g., Lazendic et al. 2006; DeLaney et al. 2006), such a data set would also permit investigation of Cas A's ejecta structure across low $\left(0.1-1 \mathrm{~cm}^{-3}\right)$ and high $\left(10^{3} \mathrm{~cm}^{-3}\right)$ density and hot $\left(10^{6}-10^{8} \mathrm{~K}\right)$ and cold $\left(10^{2} \mathrm{~K}\right)$ temperature regimes.

We thank Roger Chevalier and Jon Blondin for helpful discussions on the properties of the jet and other outlying ejecta in Cas A, Jordan Zastrow for help with cross-correlating knot IDs, and an anonymous referee for helpful comments. This work was supported by NASA through grants GO-9890 and GO-10286 from the Space Telescope Science Institute.

Akiyama, S., Wheeler, J. C., Meier, D. L., \& Lichtenstadt, I. 2003, ApJ, 584,

\section{REFERENCES}

954

Baade, W., \& Minkowski, R. 1954, ApJ, 119, 206

Bertin, E., \& Arnouts, S. 1996, A\&AS, 117, 393

Blondin, J. M., Lundqvist, P., \& Chevalier, R. A. 1996, ApJ, 472, 257

Boffi, F. R., et al. 2003, ACS Instrument Handbook, Version 4.0 (Balitmore: STScI)

Burrows, A., Hayes, J., \& Fryxell, B. A. 1995, ApJ, 450, 830

Burrows, A., Walder, R., Ott, C. D., \& Livne, E. 2005, in ASP Conf. Ser. 332, The Fate of the Most Massive Stars (San Francisco: ASP), 358

Chevalier, R. A., \& Kirshner, R. P. 1978, ApJ, 219, 931 1979, ApJ, 233, 154

DeLaney, T., \& Rudnick, L. 2003, ApJ, 589, 818

DeLaney, T., Smith, J., Rudnick, L., Ennis, J., Rho, J., Reach, W., Kozasa, T., \& Gomez, H. 2006, BAAS, 38, 139

Ennis, J. A., Rudnick, L., Reach, W. T., Smith, J. D., Rho, J., DeLaney, T., Gomez, H., \& Kozasa, T. 2006, ApJ, 652, 376

Fesen, R. A. 2001, ApJS, 133, 161

Fesen, R. A., \& Becker, R. H. 1991, ApJ, 371, 621

Fesen, R. A., Becker, R. H., \& Blair, W. P. 1987, ApJ, 313, 378

Fesen, R. A., \& Gunderson, K. S. 1996, ApJ, 470, 967

Fesen, R. A., et al. 2006a, ApJ, 636, 859 2006b, ApJ, 646, 283

Ford, H. C., et al. 1998, Proc. SPIE, 3356, 234

Gotthelf, E. V., Koralesky, B., Rudnick, L., Jones, T. W., Hwang, U., \& Petre, R. 2001, ApJ, 552, L39

Hines, D. C., et al. 2004, ApJS, 154, 290

Höflich, P., Khokhlov, A., \& Wang, L. 2001, in AIP Conf. Proc. 586, 20th Texas Symposium on Relativistic Astrophysics (Melville: AIP), 459

Höflich, P., Wheeler, J. C., \& Wang, L. 1999, ApJ, 521, 179

Hurford, A. P., \& Fesen, R. A. 1996, ApJ, 469, 246

Hwang, U., \& Laming, J. M. 2003, ApJ, 597, 362

Hwang, U., et al. 2004, ApJ, 615, L117

Kamper, K., \& van den Bergh, S. 1976, ApJS, 32, 351

Kamper, K. W., \& van den Bergh, S. 1983, in IAU Symp. 101, Supernova Remnants and their X-ray Emission, ed. J. Danziger \& P. Gorenstein (Dordrecht: Reidel), 55

Khokhlov, A. M., Höflich, P. A., Oran, E. S., Wheeler, J. C., Wang, L., \& Chtchelkanova, A. Y. 1999, ApJ, 524, L107

Kifonidis, K., Plewa, T., Janka, H.-T., \& Müller, E. 2003, A\&A, 408, 621

Kifonidis, K., Plewa, T., Scheck, L., Janka, H.-T., \& Müller, E. 2006, A\&A, 453,661

Lazendic, J. S., Dewey, D., Schulz, N. S., \& Canizares, C. R. 2006, ApJ, 651, 250

Leonard, D. C., \& Filippenko, A. V. 2001, PASP, 113, 920

Leonard, D. C., Filippenko, A. V., Ardila, D. R., \& Brotherton, M. S. 2001, ApJ, 553, 861

Leonard, D. C., Filippenko, A. V., Barth, A. J., \& Matheson, T. 2000, ApJ, 536, 239

Mazzali, P. A., et al. 2005, Science, 308, 1284

Minkowski, R. 1957, IAU Symp. 4, Radio Astronomy (Cambridge: Cambridge Univ. Press), 107

1968, in Nebulae and Interstellar Matter, ed. B. M. Middlehurst \& L. H. Aller (Chicago: Univ. Chicago Press), 623

Pavlovsky, C., et al. 2004, ACS Instrument Handbook, Version 5.0 (Baltimore: STScI)

Reed, J. E., Hester, J. J., Fabian, A. C., \& Winkler, P. F. 1995, ApJ, 440, 706

Reynoso, E. M., \& Goss, W. M. 2002, ApJ, 575, 871

Sirianni, M., et al. 2005, PASP, 117, 1049

Tananbaum, H. 1999, IAU Circ., 7246, 1

Thorstensen, J. R., Fesen, R. A., \& van den Bergh, S. 2001, AJ, 122, 297

van den Bergh, S. 1971, ApJ, 165, 457

van den Bergh, S., \& Dodd, W. W. 1970, ApJ, 162, 485

van den Bergh, S., \& Kamper, K. W. 1985, ApJ, 293, 537

Wang, L., Howell, D. A., Höflich, P., \& Wheeler, J. C. 2001, ApJ, 550, 1030

Wang, L., Wheeler, J. C., Li, Z., \& Clocchiatti, A. 1996, ApJ, 467, 435

Wheeler, J. C., Maund, J. R., \& Couch, S. M. 2008, ApJ, 677, 1091

Wilson, J. R., Mathews, G. J., \& Dalhed, H. E. 2005, ApJ, 628, 335

Winkler, P. F., Roberts, P. F., \& Kirshner, R. P. 1991, in Supernovae: The Tenth

Santa Cruz Summer Workshop in Astronomy and Astrophysics, ed. S. E.

Woosley (New York: Springer), 652

Yamasaki, T., \& Yamada, S. 2005, ApJ, 623, 1000 